\title{
The Application of Novel Nano-Thermal and Imaging \\ Techniques for Monitoring Drug Microstructure and Distribution within PLGA Microspheres
}

Fan Yang ${ }^{\mathrm{a}}$, De Chen ${ }^{\mathrm{a}}$, Zhe-fei Guo ${ }^{\mathrm{c}}$, Yong-ming Zhang ${ }^{\mathrm{c}}$, Yi Liu ${ }^{\mathrm{b}}$, Sean Askin ${ }^{\mathrm{d}}$, Duncan Q. M. Craig ${ }^{\mathrm{d}}$, Min Zhaod, e,*

a Department of Pharmacy, Guangdong Pharmaceutical University, Guangzhou 510006, China

b Department of Chemistry and Chemical Engineering, Guangdong Pharmaceutical University, Guangzhou 510006, China

c Department of Pharmacy, The Third Affiliated Hospital, Sun Yat-Sen University, Guangzhou 510000, China

d UCL School of Pharmacy, 29-39 Brunswick Square, London WC1N 1AX, UK

e Queen's University Belfast School of Pharmacy, 97 Lisburn Road, Belfast BT9 7BL, UK

Corresponding author: Min Zhao: m.zhao@qub.ac.uk 


\begin{abstract}
Poly (d,1-lactic-co-glycolic) acid (PLGA) based microspheres have been extensively used as controlled drug release systems. However, the burst effect has been a persistent issue associated with such systems, especially for those prepared by the double emulsion technique. An effective approach to preventing the burst effect and achieving a more ideal drug release profile is to improve the drug distribution within the polymeric matrix. Therefore, it is of great importance to establish a rapid and robust tool for screening and optimizing the drug distribution during pre-formulation. Transition Temperature Microscopy (TTM), a novel nano-thermal and imaging technique, is an extension of nano-thermal analysis (nano-TA) whereby a transition temperature is detected at a localized region of a sample and then designated a color based on a particular temperature/color palette, finally resulting in a coded map based on transition temperatures detected by carrying out a series of nanoTA measurements across the surface of the sample. In this study, we investigate the feasibility of applying the aforementioned technique combined with other thermal, imaging and structural techniques for monitoring the drug microstructure and spatial distribution within bovine serum albumin (BSA) loaded and nimodipine loaded PLGA microspheres, with a view to better predicting the in vitro drug release performance.
\end{abstract}

\title{
Keywords
}

Transition temperature microscopy; Microsphere; Bovine serum albumin; Nimodipine; Poly (d,1-lactic-co-glycolic) acid; Controlled drug release

\section{Introduction}

In recent years microspheres fabricated with biodegradable materials, such as poly (d,1-lactic-co-glycolic) acid (PLGA), have attracted significant attention [1-3]. 
Microspheres have been developed to encapsulate different classes of drugs including biopharmaceuticals [4,5], small molecules [6,7] and natural products [8,9]. The main driving force for developing such microspheres has typically been to prolong the duration of action of the incorporated drugs. In addition, microsphere formulations have also been shown to possess other advantages, including site-specific release with a concomitant reduction in toxicity $[10,11]$. However, there are still limitations regarding drug release from polymeric microspheres that remain unresolved; these include burst release effects, low drug release rates (and hence concentrations) at later stages and incomplete release due to drug entrapment within the microspheres. Among these, the burst release effect is the most problematic issue, particularly for water-soluble drugs [12]. In order to understand the mechanisms underpinning burst release, and to minimize such effects, the drug distribution and microstructure within the polymeric matrix should be more fully understood.

The investigation of drug uptake and distribution within microspheres has focused largely on the measurement of the drug loading using a range of assay methods. The content of small molecule drugs encapsulated in microspheres is usually measured by titration, high performance liquid chromatography (HPLC) or UV spectrophotometry, while the Kjeldahl, Folin-phenol or bicinchoninic acid (BCA) methods [13] are used to determine the loading of peptide or protein therapeutics. Other analytical techniques that are routinely used to characterize microsphere formulations include modulated temperature differential scanning calorimetry (MTDSC) to study the amorphous properties of the microspheres, X-ray diffraction (XRD) [14] to study the crystallinity and Fourier transform infrared spectroscopy (FTIR) [15] to study the chemical composition and interactions within the systems.

The characterization methods routinely used to analyze microspheres have some common limitations in that they interrogate the bulk sample rather than individual microspheres, while such methods also do not provide information regarding the spatial distribution of the drug within the system. In addition, traditional drug loading/ encapsulation measurements are normally lengthy and complex, hence it is of 
considerable interest to establish a rapid and robust analytical platform for fast evaluating the drug content and spatial distribution within the microspheres.

Transition temperature microscopy (TTM) is a novel technique for automated analysis of the local temperature transitions over an area of a sample surface. Typically, thermal analysis methods such as differential scanning calorimetry (DSC) are used to measure thermal transitions such as the melting point $\left(\mathrm{T}_{\mathrm{m}}\right)$ of crystalline materials and the glass transition temperature $\left(\mathrm{T}_{\mathrm{g}}\right)$ of amorphous samples. This technique, however, is usually performed on bulk samples and therefore the transitions measured are not spatially differentiated. For this reason, further research and development was conducted on local measurements of thermal phase transitions using heated probe tips based on Atomic Force Microscopy (AFM) [16-18]. As a result, Micro Thermal Analysis ( $\mu$ TA) and Nano Thermal Analysis (Nano TA) have been successively developed. Nevertheless, both the conventional $\mu$ TA and NanoTA measurements are carried out manually at single points or a handful of points without the ability to automatically resolve special variations in thermal transition temperatures. TTM is a recent extension of NanoTA whereby transition temperatures are detected at a series of points in a defined region of a sample; these points are then color-coded based on the transition temperature, resulting in a map based on transition temperatures detected across the surface of the sample [19-20]. This method therefore has the potential to determine the distribution of the drug and polymer across the surface and cross section of a single microsphere.

In this study, nimodipine, a hydrophobic drug with a molecular weight of 418 , and bovine serum albumin (BSA), a hydrophilic protein with a molecular weight of approximately $68 \mathrm{KDa}$, were selected as small and large molecular weight drug models. Nimodipine-PLGA microspheres and BSA-PLGA microspheres were prepared with three levels of drug loading. The morphology and in vitro release of the different microsphere formulations were then studied. Additionally, TTM was used to study the drug distribution on the surfaces and on the internal cross-sections of single microspheres. Finally, modulated temperature differential scanning calorimetry (MTDSC), X-ray diffraction (XRD) and Fourier transform infrared spectroscopy 
(FTIR) were also used to analyse the bulk properties of the systems. By combining the above methods, the drug distribution and microstructure within the different types of microspheres could be interpreted more comprehensively. The study therefore explores the utility of TTM as a novel tool to provide a more detailed understanding of spatial distribution of drugs in microspheres, with a view to providing insights into the relationship between such distribution and in vitro release.

\section{Materials and Methods}

\subsection{Materials}

Bovine serum albumin (BSA) was purchased from American Genview Co. (USA). Nimodipine was purchased from Zhuhai Yuan-Cheng Med-chemical Co. Ltd. (Zhuhai, China). The PLGA (Lactide:Glycolide 50:50) was obtained from Absorbable Polymers International (Birmingham, USA). Polyvinyl alcohol-124 (PVA) and Poloxamer (F68) were obtained from Shantou Chemical Regents Co. (Shantou, China). Tween 80 was purchased from BASF (Ludwigshafen, Germany). Dichloromethane and sodium chloride were obtained from Guangzhou Chemical Regents Co. (Guangzhou, China).

\subsection{Preparation of Microspheres}

Preparation of nimodipine-PLGA microspheres (nimodipine-PLGA) using a membrane emulsification method

PLGA (15\%, w/v) and nimodipine with three different loadings (see Table 1) were firstly dissolved in $5 \mathrm{~mL}$ dichloromethane to form the dispersed phase, which was then added to the Shirasu Porous Glass (SPG) membrane emulsification machine and extruded through the SPG membrane under $\mathrm{N}_{2}$ pressure to achieve uniform-sized droplets; 200ml PVA solution $(1 \%, \mathrm{w} / \mathrm{v})$ was used as the continuous phase. The mix was further stirred at $250 \mathrm{rpm}$ for $4 \mathrm{hr}$ at room temperature after which the majority of dispersed phase was dispersed into the continuous phase and emulsion drops solidified following dichloromethane evaporation. Microspheres were then washed with de-ionized water and finally collected after filtration and a $24 \mathrm{hr}$ drying. 
Preparation of BSA-PLGA microspheres (BSA-PLGA-MS) using a double emulsion-solvent evaporation method

PLGA $(15 \%, \mathrm{w} / \mathrm{v})$ was dissolved in dichloromethane to form the organic phase. BSA with three different loadings (see Table 1) was dissolved in a F68 solution (1\%, $\mathrm{w} / \mathrm{v}$ ) to form the internal aqueous phase, which was then added to organic phase while stirring at a suitable temperature and speed to form the initial W/O emulsion. The W/ O emulsion was then added to PVA solution (external aqueous phase) while stirring for $10 \mathrm{~min}$ to form the double emulsion and then transferred to a PVA solution while stirring slowly to solidify the microspheres by extraction, which were then washed prior to freeze drying.

\subsection{Determination of Drug Loading and Encapsulation Efficiency}

Nimodipine-loaded microspheres $(20 \mathrm{mg})$ were dissolved completely in $1 \mathrm{~mL}$ dichloromethane and then diluted 100-fold with ethanol in a $10 \mathrm{~mL}$ volumetric flask, followed by centrifugation under $10,000 \mathrm{rpm}$ for 20 minutes. Finally the nimodipine concentration in the supernatant was determined spectrophotometrically $(356 \mathrm{~nm})$.

BSA-loaded microspheres $(10 \mathrm{mg})$ were suspended in $5 \mathrm{~mL}$ SDS solution $(5 \%$ w/v) with $0.1 \mathrm{M} \mathrm{NaOH}$. The suspension was then shaken in the water bath at $37^{\circ} \mathrm{C}$ for $48 \mathrm{~h}$ to ensure the microspheres were completely dissolved. The supernatant was extracted after centrifugation, and the protein content was determined using the Bicinchoninic Acid (BCA) method.

\subsection{Measurement of Particle Size and Size Distribution}

Particle size and size distribution of the microspheres were measured using a laser diffraction particle size analyzer (Counter LS130, USA) using water as the dispersion medium. 


\subsection{Scanning Electron Microscopy (SEM)}

The morphology of both the microsphere surfaces and the cross-sections were imaged by scanning electron microscopy (SEM) (Hitachi S-4800, Japan) after being coated with gold in an argon atmosphere. To section the particles, the microspheres were firstly suspended in two different embedding agents: optimal cutting temperature (OCT) compound (Shanghai solarbio Bioscience \& Technology Co., LTD, China), and an aqua solution containing gelatin and glycerin prior to solidification via being stored at $-20^{\circ} \mathrm{C}$. The fixed samples were then frozen in liquid nitrogen for $5 \mathrm{~min}$ and cryosectioned under $-40^{\circ} \mathrm{Cbased}$ on an established method [21].

\subsection{In vitro Drug Release Measurement}

Prior to the in vitro release test, $5 \mathrm{~mL}$ solution of nimodipine in phosphate buffered saline (PBS, pH7.4) solution (containing 0.5\% w/v SDS) was placed in a dialysis bag which was submerged in $195 \mathrm{ml}$ PBS solution in a constant temperature oscillator held at $37{ }^{\circ} \mathrm{C} \pm 0.2{ }^{\circ} \mathrm{C}$ with $100 \mathrm{rpm}$ rotation speed. The concentration of nimodipine inside or external to the dialysis bags was found to be the same after $2 \mathrm{hr}$, indicating the dialysis bag itself did not affect the drug transport.

Nimodipine-loaded microspheres $(30 \mathrm{mg})$ were placed in a dialysis bag, and 5 $\mathrm{mL}$ phosphate buffered saline (PBS, $\mathrm{pH} 7.4$ ) solution (containing $0.5 \% \mathrm{w} / \mathrm{v}$ SDS) added to disperse the microspheres. The dialysis bag was then submerged in PBS solution $(195 \mathrm{~mL})$, and the in vitro release test was conducted in a constant temperature oscillator at $37{ }^{\circ} \mathrm{C} \pm 0.2{ }^{\circ} \mathrm{C}$ and $100 \mathrm{rpm}$ rotational speed. At predetermined time points, $5 \mathrm{~mL}$ of the release medium was sampled and fresh medium added to maintain a constant total volume. The amount of drug released at each time point was quantified by UV spectrophotometry using a standard curve.

BSA-loaded spheres $(50 \mathrm{mg})$ were placed in a centrifuge tube and PBS solution $(1.5 \mathrm{~mL})$ was added. The centrifuge tube was then put in a water bath oscillator to conduct the in vitro release test at $37^{\circ} \mathrm{C} \pm 0.2{ }^{\circ} \mathrm{C}$ and $100 \mathrm{rpm}$ rotational speed. At each time point, the sample was centrifuged, and the supernatant was sampled to 
determine the protein content using the BCA method. Fresh release medium was added in the centrifuge tube to continue the release experiment.

\subsection{X-ray Diffraction (XRD)}

XRD (Bruker D8 Advance, Germany) measurements were carried out at a voltage of $40 \mathrm{kV}$ and a current of $40 \mathrm{~mA}$. Data was collected at a scan rate of $0.02 \%$ 0.15 over a range of 5 to $50^{\circ} 2 \theta$.

\subsection{Fourier Transform Infrared (FTIR) Spectroscopy}

The raw materials of nimodipine and BSA, and blank and drug-loaded microspheres were mixed with potassium bromide $(\mathrm{KBr})$ respectively, ground and tableted. The scan range was from 4000 to $400 \mathrm{~cm}^{-1}$.

\subsection{Modulated Temperature Differential Scanning Calorimetry (MTDSC)}

MTDSC (Q1000 TA Instruments, Delaware, USA) measurements were carried out at $2{ }^{\circ} \mathrm{C} / \mathrm{min}$ with a modulation of $\pm 0.212^{\circ} \mathrm{C}$ for every $40 \mathrm{~s}$ over a temperature range of 0 to $250{ }^{\circ} \mathrm{C}$ using standard pans. The instrument was calibrated with indium, tin and octadecane before use.

\subsection{Transition Temperature Microscopy (TTM)}

TTM was performed on both raw materials and microspheres using a VESTA TM system equipped with an AN-200 ThermaLever probe, both from Anasys Instruments (Santa Barbara, USA). With raw materials in loose powder, a mini tablet sample was made to ensure a flat and smooth surface for TTM measurement. To investigate the cross section of the microspheres, samples were sectioned using the same method as stated in 2.5. The voltage was calibrated to temperature by carrying out LTA measurements on three standard polymeric samples with known melting points. The experiments were carried out from room temperature at a heating rate of $10^{\circ} \mathrm{C} / \mathrm{s}$. For each sample, an area of $50 \times 50 \mu \mathrm{m}$ was analysed. 


\section{Results}

\subsection{Measurement of particle size, drug loading and encapsulation efficiency}

The drug loading and encapsulation efficiencies of the different microsphere formulations were measured and are listed in Table 1. With both model drugs, the lowest drug loading resulted in a relatively high encapsulation efficiency while the particle size increased with an increase in drug loading. It is noted that the particle size with a general distribution of $65-100 \mu \mathrm{m}$ did not vary significantly among different formulations, making the later analysis of drug release profiles less complicated.

Table 1. Particle size, drug loading and encapsulation efficiency measured for BSA and nimodipine microspheres.

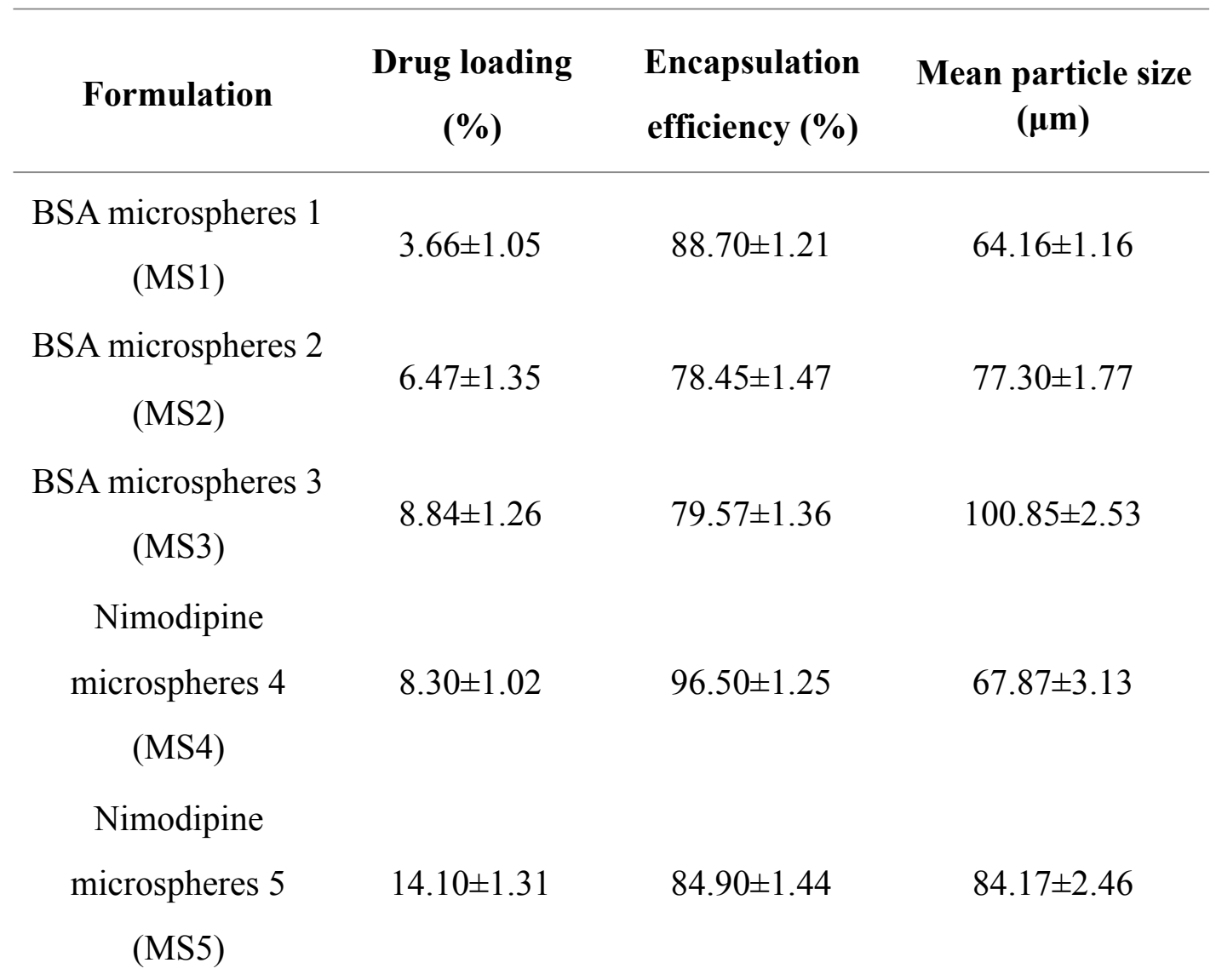


Nimodipine

microspheres 6

\subsection{SEM results}

SEM showed that the surfaces of BSA-loaded microspheres (Figure 1 a-c) were relatively smooth compared to that of nimodipine systems (Figure 1d-f), with the latter showing some evidence for the presence of surface crystallites at higher loadings. For the cross-sections, BSA-loaded microspheres showed a considerable amount of internal cavities while the nimodipine-PLGA microspheres showed a much smoother internal morphology, with only the highest drug loading appeared to present cavities. The difference between the two model drug systems in both the surface and cross section morphology was partially due to the different methods of preparation applied and partially due to the different properties of the two model drugs. Nimodipine is a lipophilic drug and is expected to have good miscibility with PLGA, hence it is quite likely for such a drug to be molecularly dispersed in the PLGA matrix. The fact that both components were dissolved in a single disperse phase during the preparation may explain why the cross-sections of nimodipine microspheres did not exhibit as many cavities as BSA microspheres did. Likewise the crystals on the surface may be caused by recrystallization of a small amount of nimodipine during the evaporation of the organic solvent, leading to crystallite adhesion to the surface of the microspheres.

When preparing the BSA microspheres with the double emulsion solvent evaporation method, the hydrophilic protein and PLGA, a lipophilic polymer, were dissolved in distinct aqueous and organic solvents, respectively. During the solidification process, BSA could not be mixed with PLGA and remained dispersed in the solid PLGA particles within aqueous droplets due to the significant solubility difference between the two components and the lack of miscibility between the two 
solvent systems used. Finally, after the microspheres were freeze-dried, the aqueous solvent was removed and the cavities seen in the cross sections were formed.

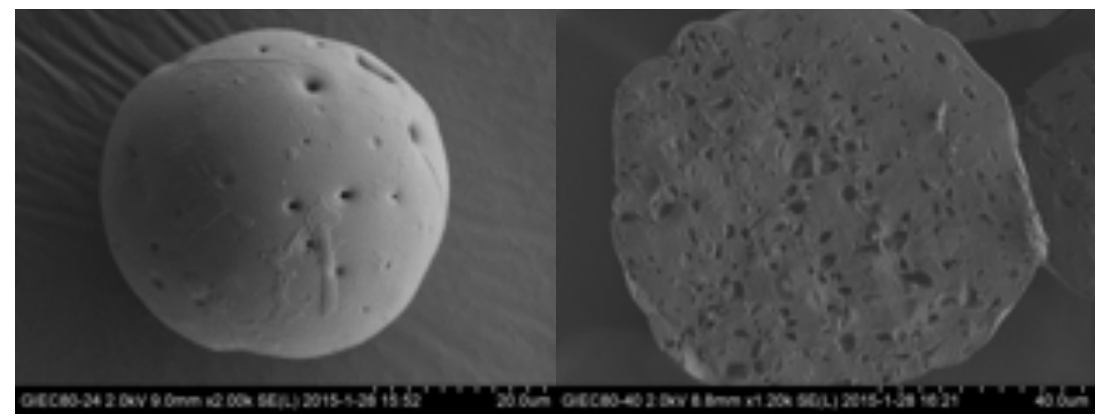

a. Microsphere 1: 3.66\% BSA loaded PLGA microspheres

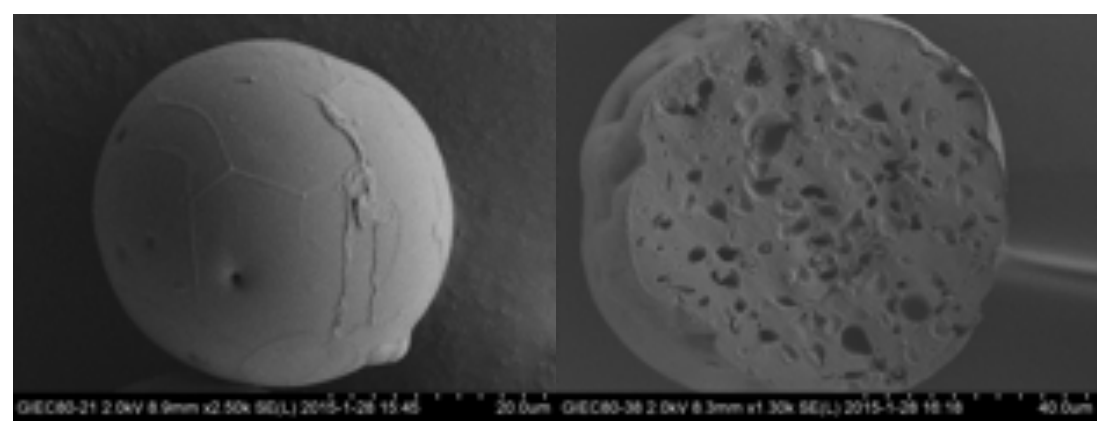

b. Microsphere 2: 6.47\% BSA loaded PLGA microspheres

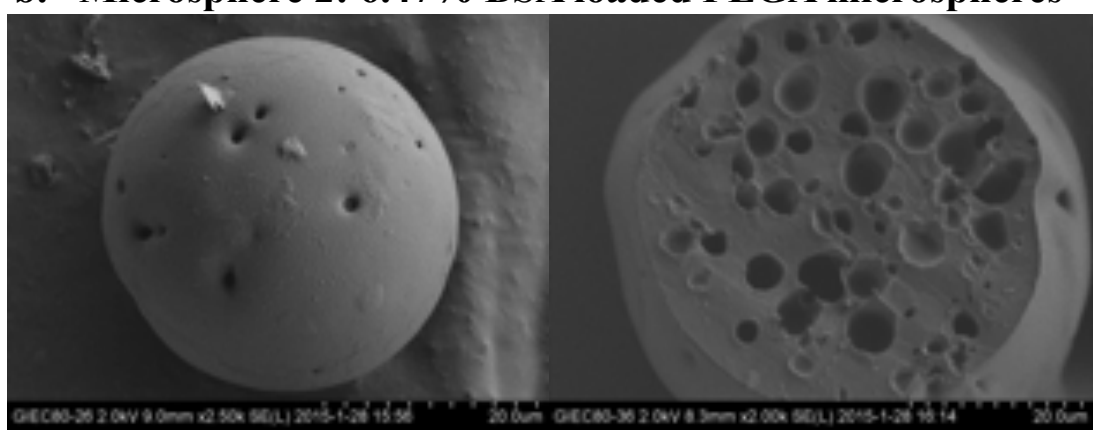

c. Microsphere 3: 8.84\% BSA loaded PLGA microspheres

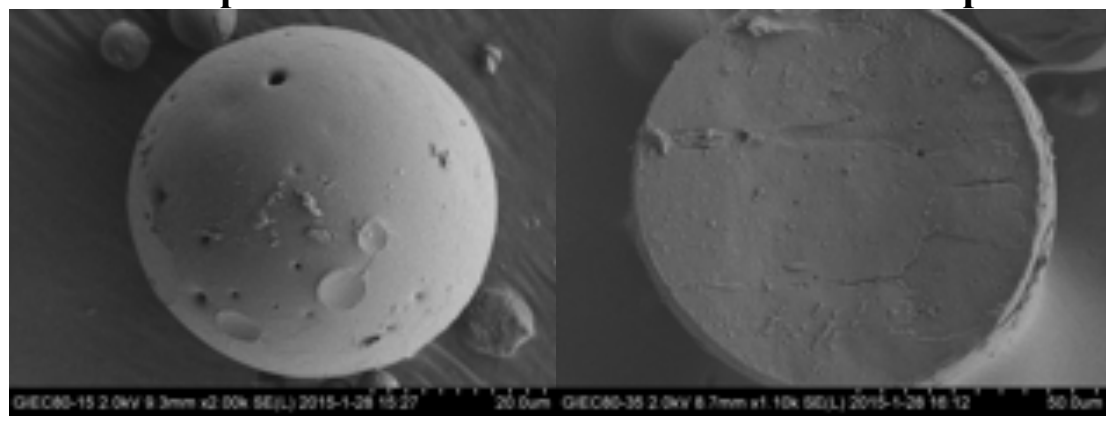

d. Microsphere 4: 8.30\% nimodipine loaded PLGA microspheres 


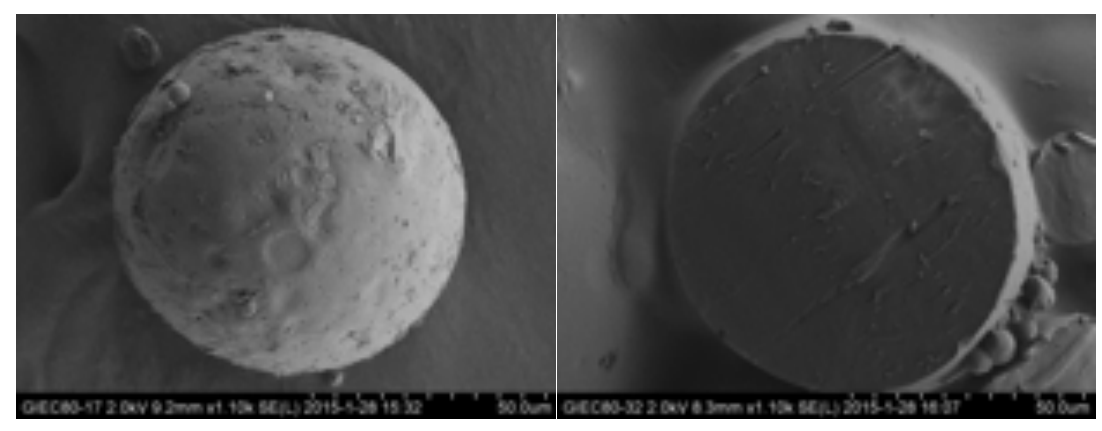

e. Microsphere 5: $14.10 \%$ nimodipine loaded PLGA microspheres

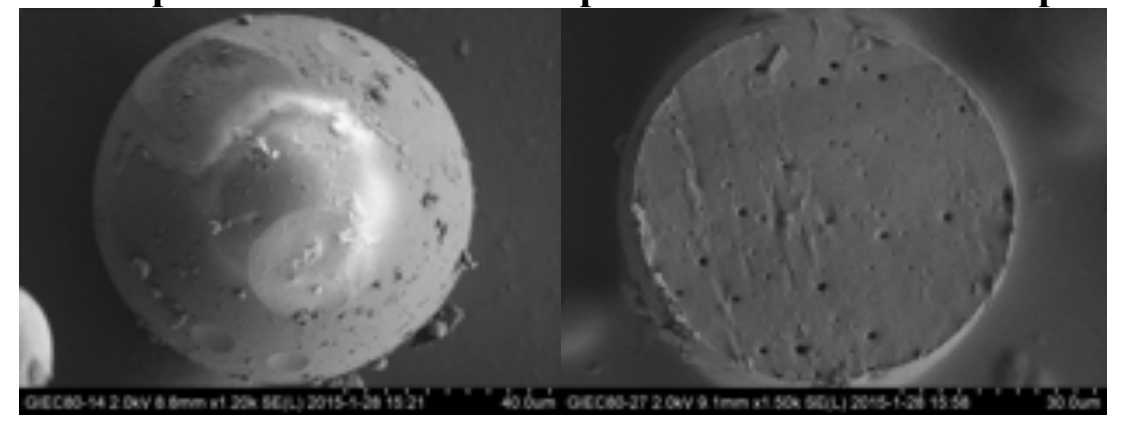

f. Microsphere 6: $\mathbf{2 2 . 8 0 \%}$ nimodipine loaded PLGA microspheres

Figure 1: SEM images of the outer surface (left) and cross section (right) of BSA loaded PLGA microspheres (a-c) and nimodipine loaded PLGA microspheres (df) with various drug loadings.

\subsection{In vitro drug release profiles}

As shown in Figure 2, the burst release from BSA microspheres was significant with a large proportion of BSA being released during the first hour. After 40 days, there was still a considerable amount of drug that remained unreleased from the formulation. This burst release phenomenon was probably caused by the hydrophilic nature of BSA; the aqueous solubility of BSA is high, therefore when the BSA microspheres were placed in an aqueous medium for the in vitro release studies, the BSA molecules present near the surface would quickly dissolve and diffuse into the bulk release medium, which at least partially accounts for the observed burst release. There are a number of factors that may affect the burst release, including the drug solubility, the overall drug loading and the drug level on the surface of the microspheres.

In terms of the nimodipine microspheres, the drug was released at a relatively constant rate throughout the first 40 days of the in vitro release study, indicating a well sustained release profile. After 40 days, the drug was not completely released, 
although up to this point the release rate remained largely constant. The in vitro release of nimodipine from microspheres appeared to be controlled by the degradation of PLGA based on previous studies/recognition of PLGA as a biodegradable polymer for sustained release.
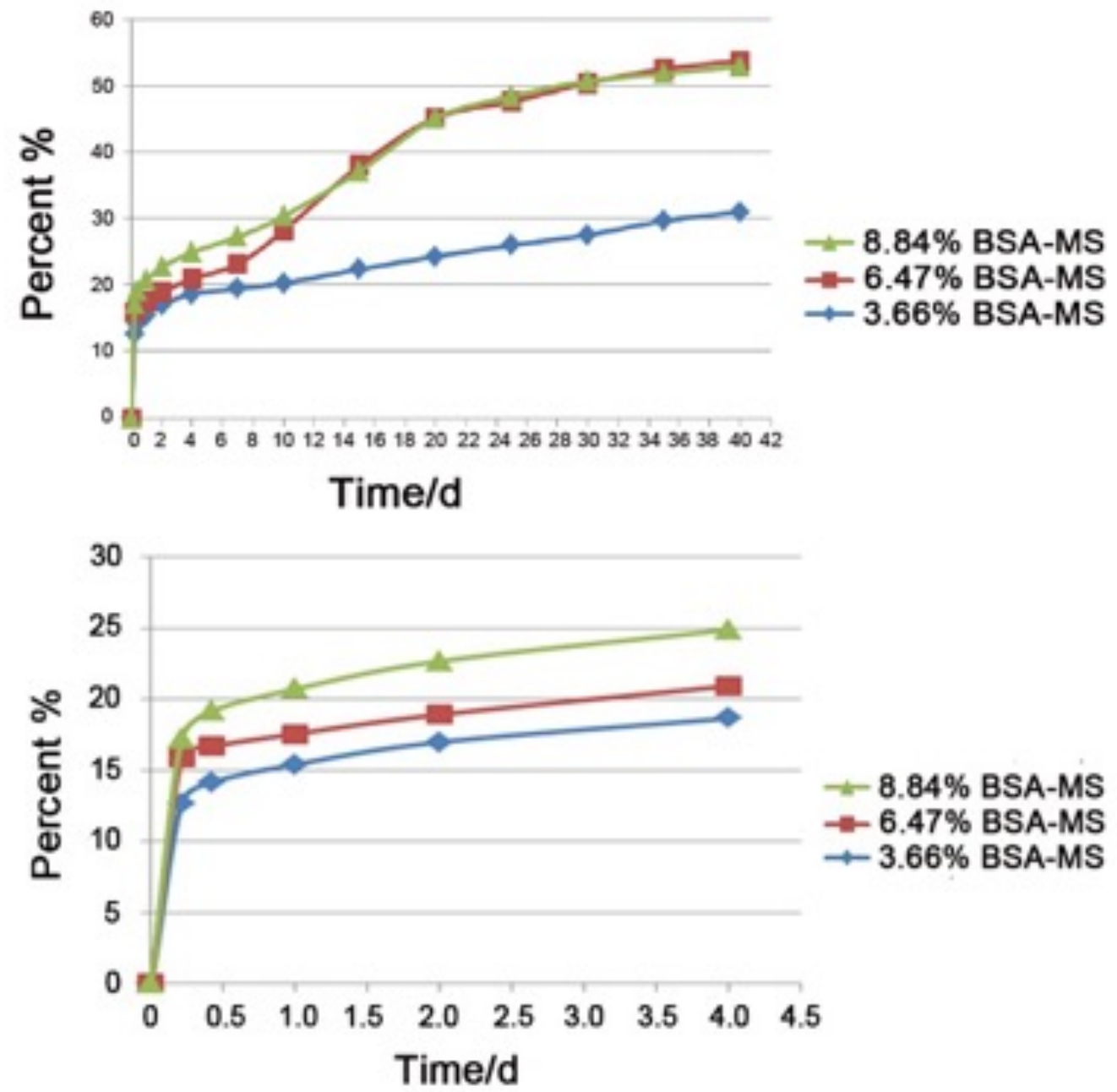

Figure 2 In vitro drug release profiles of BSA loaded PLGA microspheres, with the early stages of drug release profiles being enlarged in the lower figure 


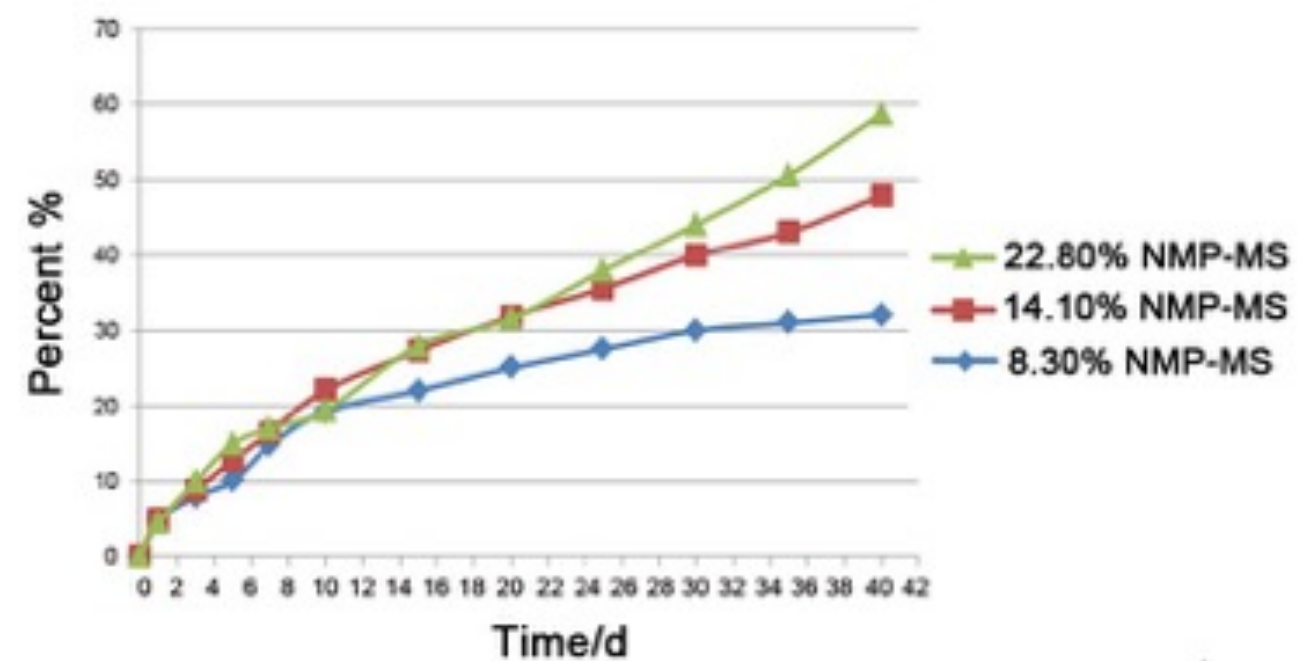

Figure 3 In vitro drug release profiles of nimodipine loaded PLGA microspheres

\subsection{XRD results}

The XRD results are shown in Figures 4 and 5. The diffractogram of BSA alone displayed a broad halo which indicated that the raw material existed in the amorphous form (Figure 4). The diffractogram corresponding to microsphere3, which had the highest BSA load of $8.8 \%$, still exhibited no intense Bragg reflections; therefore, the amorphous form persisted on entrapment in the microspheres.

Nimodipine exhibited numeroussharp and intense Bragg reflections in the diffractogram, indicating that the raw material of nimodipine existed in the crystalline form (Figure 5). In terms of the nimodipine microsphere formulations, microspheres4 and microspheres 6 (drug loadings of $8.30 \%$ and $22.80 \%$, respectively) both exhibited the characteristic Bragg reflections of the nimodipine crystals. However, the characteristic nimodipine crystalline peaks in these formulations were all very weak. 


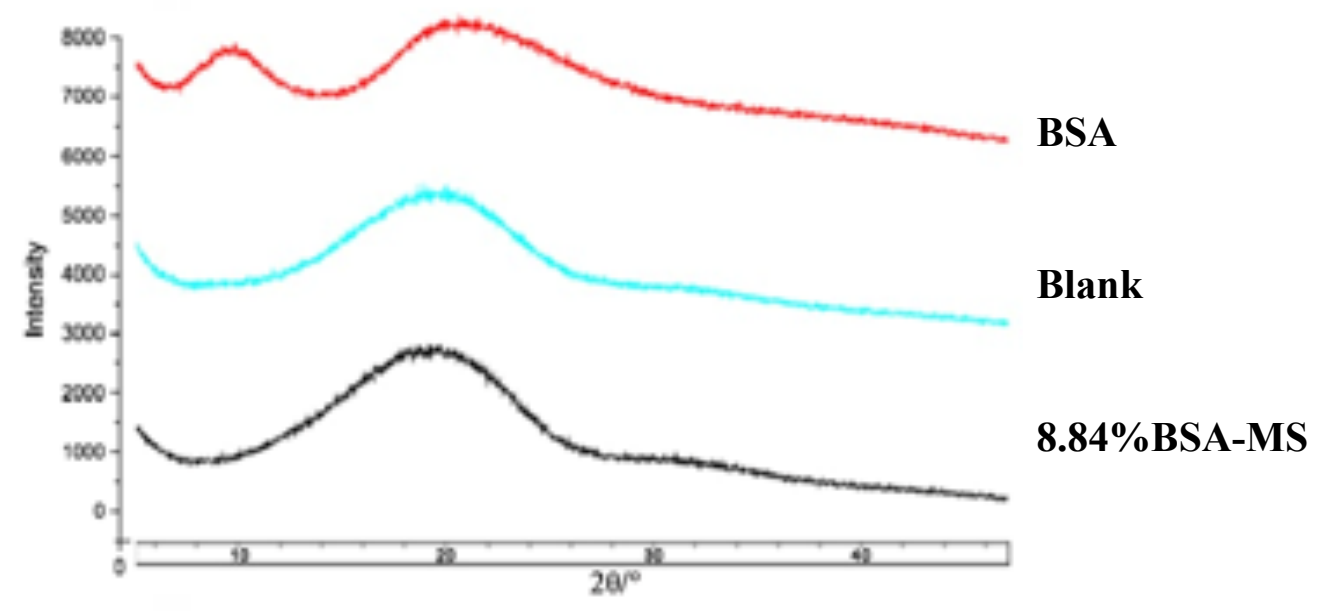

Figure 4 XRD traces of BSA loaded PLGA microspheres with the greatest drug loading (microsphere 3) and reference samples

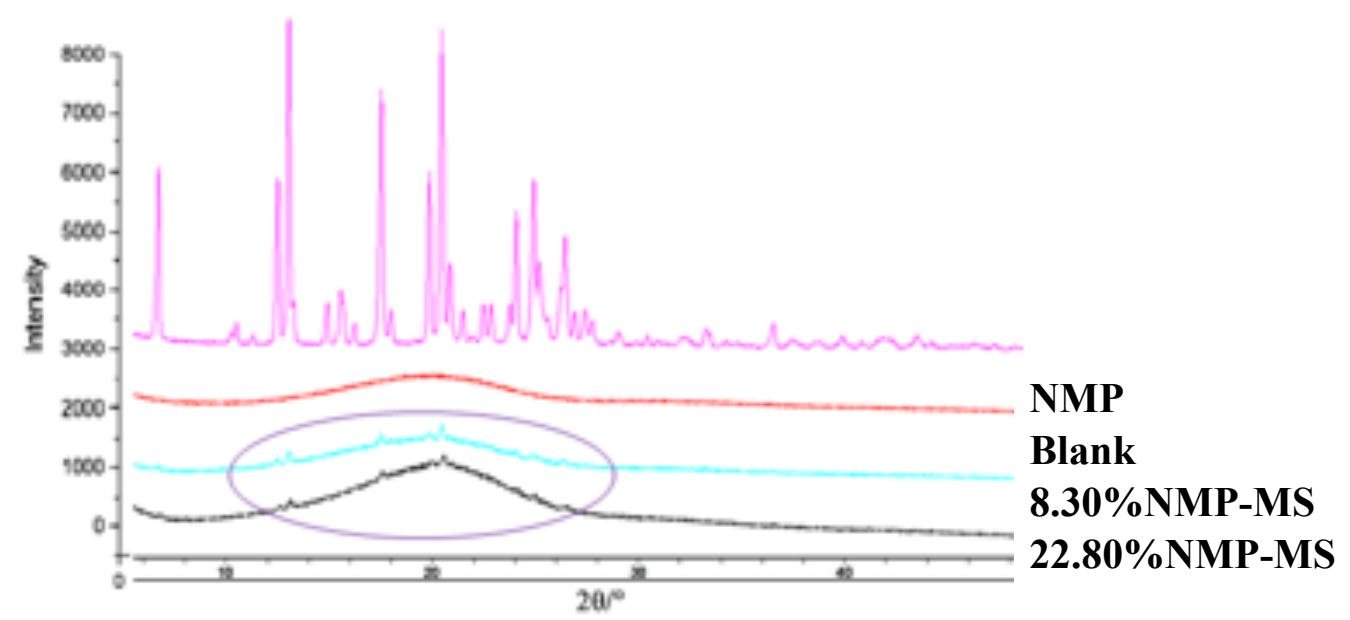

Figure 5 XRD traces of nimodipine loaded PLGA microspheres with the lowest (microsphere 4) and highest drug (microsphere 6) loadings and reference samples

\subsection{FTIR results}

Figure 6 shows the FTIR spectra of the BSA raw material, blank microspheres and BSA microspheres with different drug loading. The FTIR spectrum for the BSA raw material showed a characteristic peak of BSA at $1656 \mathrm{~cm}^{-1}$, representing the 
stretching vibration peak of $-\mathrm{C}=\mathrm{O}$ in the amide $\mathrm{I}$ band of BSA. It was found that an increase in BSA loading in the microspheres resulted in an increase in the intensity of the amide I band, which suggested enhanced BSA encapsulation in the microspheres as expected.

However, it was noteworthy that in the FTIR spectra of BSA microspheres the characteristic amide I band was red shifted to $1635,1635,1639 \mathrm{~cm}^{-1}$ for microspheres 1, 2 and 3, respectively. The characteristic peaks corresponding to the amide I band are very sensitive to changes of the secondary structures of protein. The peaks at $1650-1658 \mathrm{~cm}^{-1}$ represented the $\alpha$-helix structure while the peaks at $1620-1640 \mathrm{~cm}^{-1}$ represented the $\beta$-sheet structure. The FTIR spectra of the BSA-loaded microspheres suggested that the majority of $\alpha$-helix structure in BSA had changed to $\beta$-sheet structure after being encapsulated into microspheres. This may be due to the forming of hydrogen bonds between BSA and PLGA during encapsulation.

Figure 7 showed the FTIR spectra of the nimodipine raw material, blank microspheres and nimodipine microspheres with different drug loading. The FTIR spectrum of nimodipine raw material displayed two characteristic peaks of nimodipine at $3300 \mathrm{~cm}^{-1}$ and $1694 \mathrm{~cm}^{-1}$, which represented the stretching vibrations of $\mathrm{N}-\mathrm{H}$ and $-\mathrm{C}=\mathrm{O}$ of nimodipine, respectively. Similar to the BSA microspheres discussed earlier, the intensity of the peaks reflected the extent of encapsulation of drug in the microspheres. In terms of the peak positions for the nimodipine loaded microspheres, the position of the N-H stretching vibration blue shifted from $3300 \mathrm{~cm}^{-1}$ with the pure drug to $3390,3397,3387 \mathrm{~cm}^{-1}$ with microspheres 4,5 and 6 respectively, and the position of the $-\mathrm{C}=\mathrm{O}$ stretching vibration shifted slightly from $1694 \mathrm{~cm}^{-1}$ to $1697 \mathrm{~cm}^{-1}$ with all formulations. 


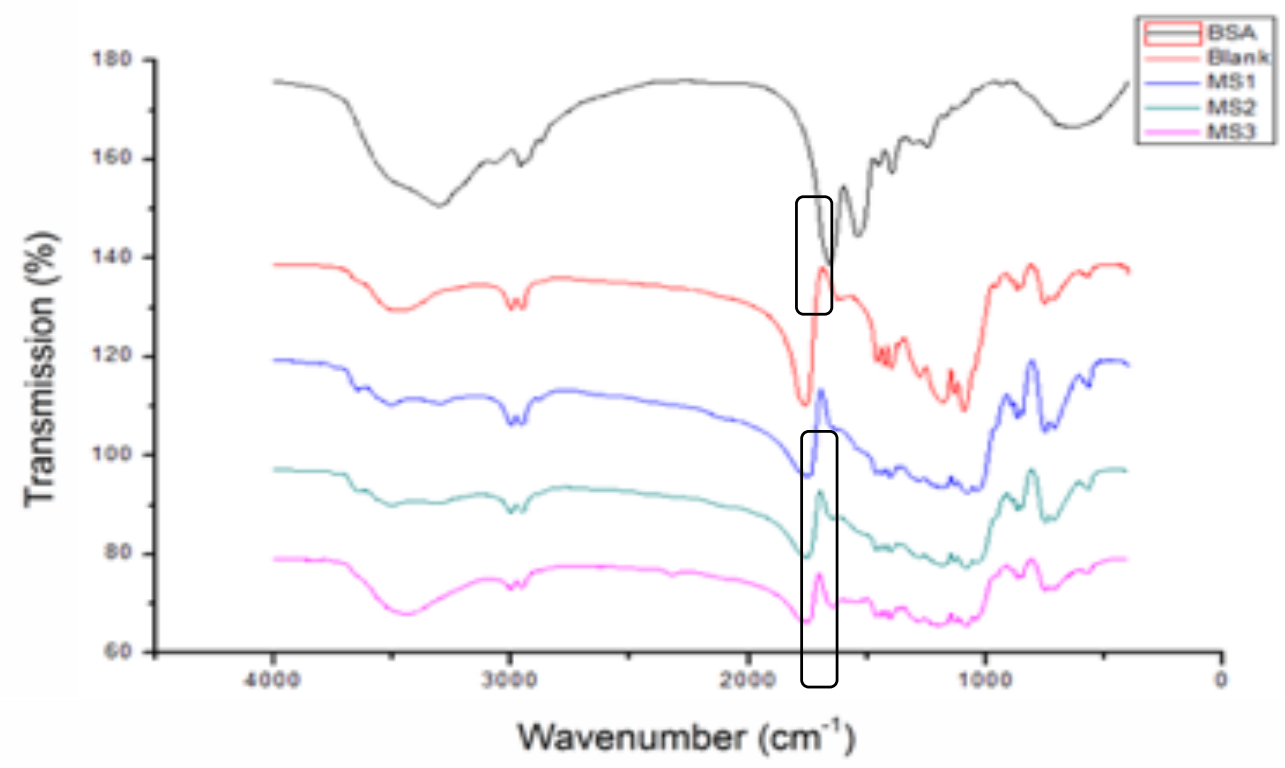

Figure 6 FTIR spectra of BSA loaded PLGA microspheres and reference samples

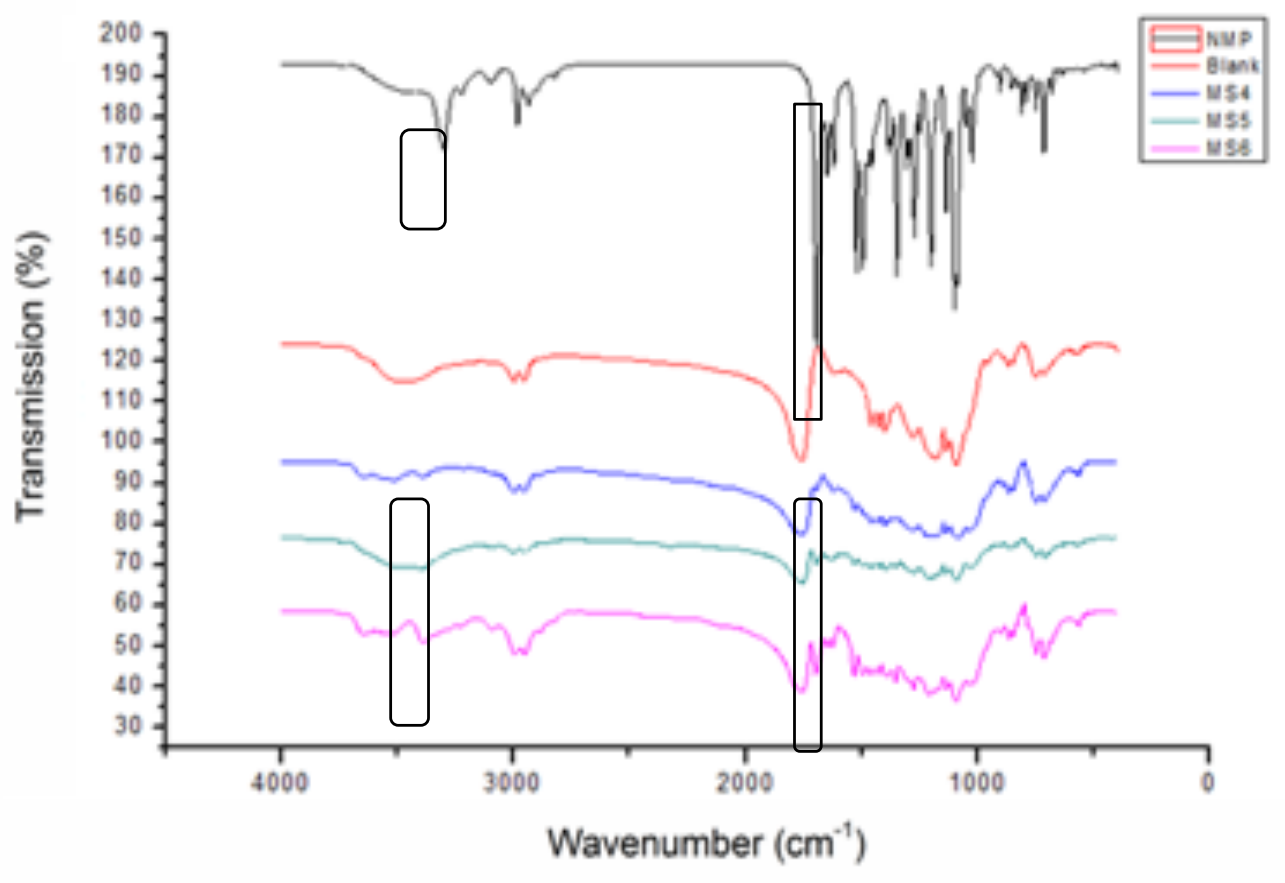

Figure 7 FTIR spectra of nimodipine loaded PLGA microspheres and reference samples

\subsection{MTDSC results}


The results of MTDSC were shown in Figures 8-10. The thermal properties of the raw materials of BSA, nimodipine and PLGA were determined first. As shown in Figure 8(a), BSA was characterized by two endothermic peaks at $62.5{ }^{\circ} \mathrm{C}$ and 215.9 ${ }^{\circ} \mathrm{C}$. Previous studies $[22,23]$ have shown that BSA experiences two phases of change during the heating process. In the first stage, occurring at a lower temperature, the $\alpha$ helix peptides may change conformation and assemble to form a random coil structure; in addition, the adjacent inverted parallel $\alpha$-helix peptides may form a $\beta$ sheet structure through hydrogen bonds during the heating process. The changes at this stage are reversible. This stage involves a plurality of structural changes between many different protein structures hence the endothermic peak looks broad. In the second stage at a higher temperature, BSA degraded and the structural changes are irreversible. Based on the previous findings above, we may reasonably ascribe the first endothermic peak of BSA measured at $62.5^{\circ} \mathrm{C}$ to a result of reversible changes and the second endothermic peak at $215.9^{\circ} \mathrm{C}$ to an irreversible degradation.

As demonstrated in Figure 8(b), the thermodynamic properties of raw nimodipine materials were easier to analyze than those of BSA, due to its smaller molecular size and simpler chemical structure. Only one narrow endothermic peak at $125.8{ }^{\circ} \mathrm{C}$, representing the melting point of the crystalline material, was present in the thermogram. In terms of PLGA raw material (Figure 8(c)), it was characterized with a single $\mathrm{T}_{\mathrm{g}}$ at about $48.1^{\circ} \mathrm{C}$, which was accompanied with an endothermic relaxation measured at $45.9^{\circ} \mathrm{C}$ in the total heat flow of the MTDSC data, indicating the pure amorphous nature of PLGA.

The MTDSC results of the BSA-PLGA microspheres with different drug loadings are shown in Figure 9. The thermograms showed that with increasing drug loading the endothermic peaks associated with BSA changed accordingly. Since both BSA and PLGA had an endothermic transition around $50{ }^{\circ} \mathrm{C}$, and the drug loadings in the BSA microspheres were relatively low, the peaks associated with BSA at about $50{ }^{\circ} \mathrm{C}$ were overshadowed by the peaks associated with PLGA, hence the thermograms mainly showed the features of the PLGA. However, since the endothermic peaks near $50{ }^{\circ} \mathrm{C}$ were the superposition of the two components, when the amount of PLGA was almost 
unchanged, the area of the peak became larger with the increase of BSA loading. The endothermic peak measured with $\mathrm{BSA}$ alone at $215.9^{\circ} \mathrm{C}$ was not present in the thermograms of the BSA-PLGA microspheres, which was likely to be due to a combination of the low drug loading and the weak nature of such a thermal transition. Further increase in BSA loading, however, did appear to result in a more visible endothermic peak.

The MTDSC results of the nimodipine-PLGA microspheres with different drug loadings were shown in Figure 10. As stated earlier, pure nimodipine presented a melting point at $125.8^{\circ} \mathrm{C}$. As for PLGA based microspheres, a 'depressed melting' was detected, indicating the compatibility/interaction (hydrogen bonding) between the two components. It was found that the higher the drug loading the greater the depressed temperature. The enthalpy of this transition also increased with drug loading; implying the nimodipine loading of microspheres had a positive correlation with the endothermic peak area. In addition, an endothermic relaxation peak was detected for all nimodipine loaded microspheres between $44-48^{\circ} \mathrm{C}$, indicating a glass transition of the amorphous phase. It was notable that no marked plasticizing effect was seen which may be due to similar glass transition temperatures between PLGA and nimodipine.

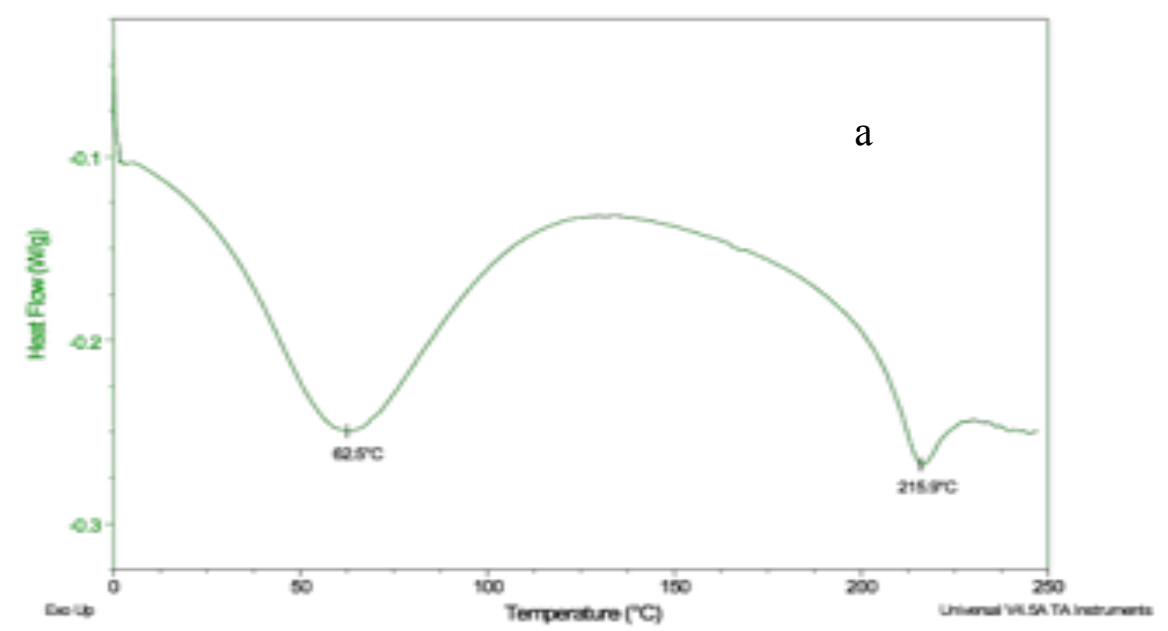



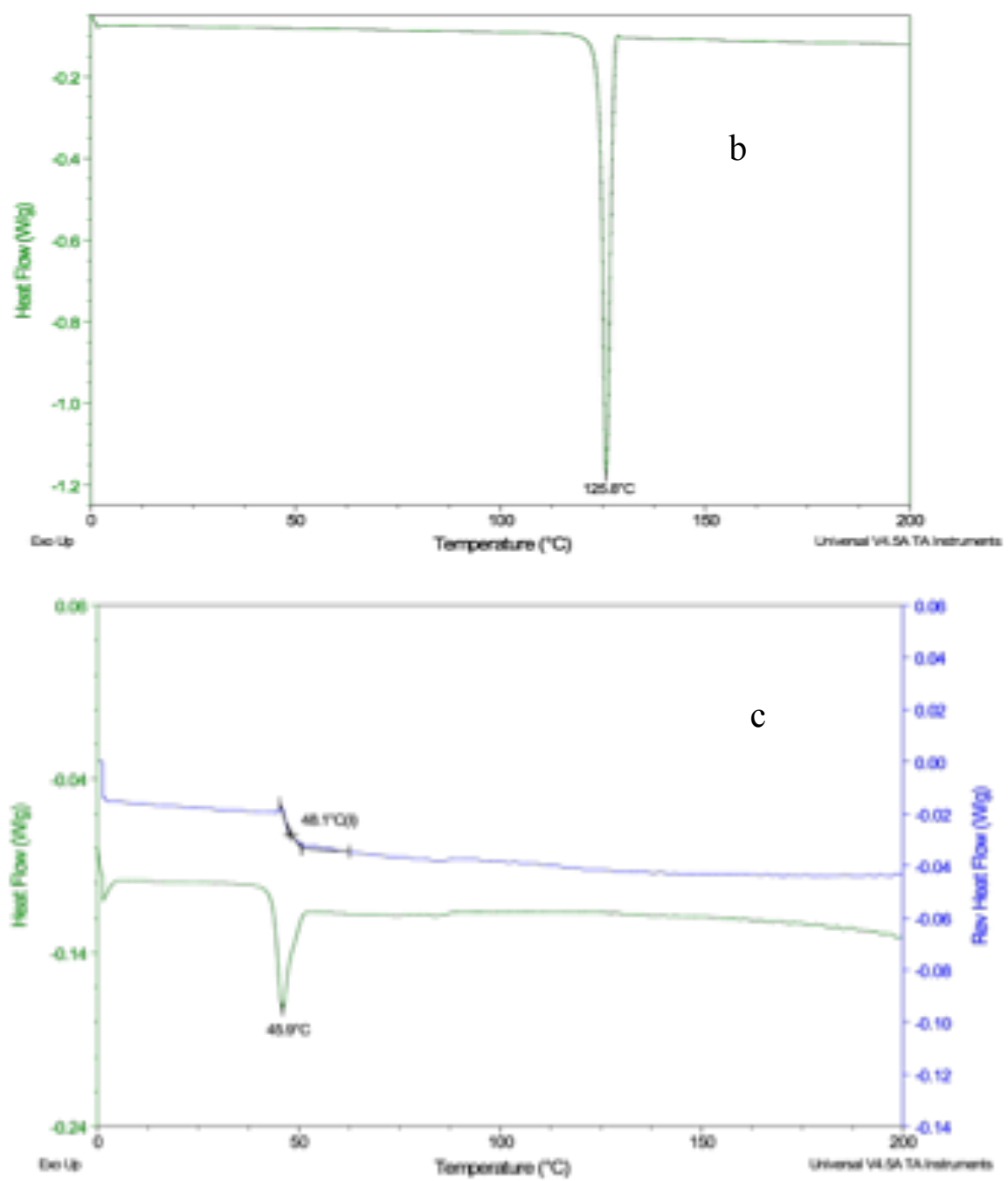

Figure 8 MTDSC heat flows of BSA (a), nimodipine (b) and PLGA (c) raw materials 


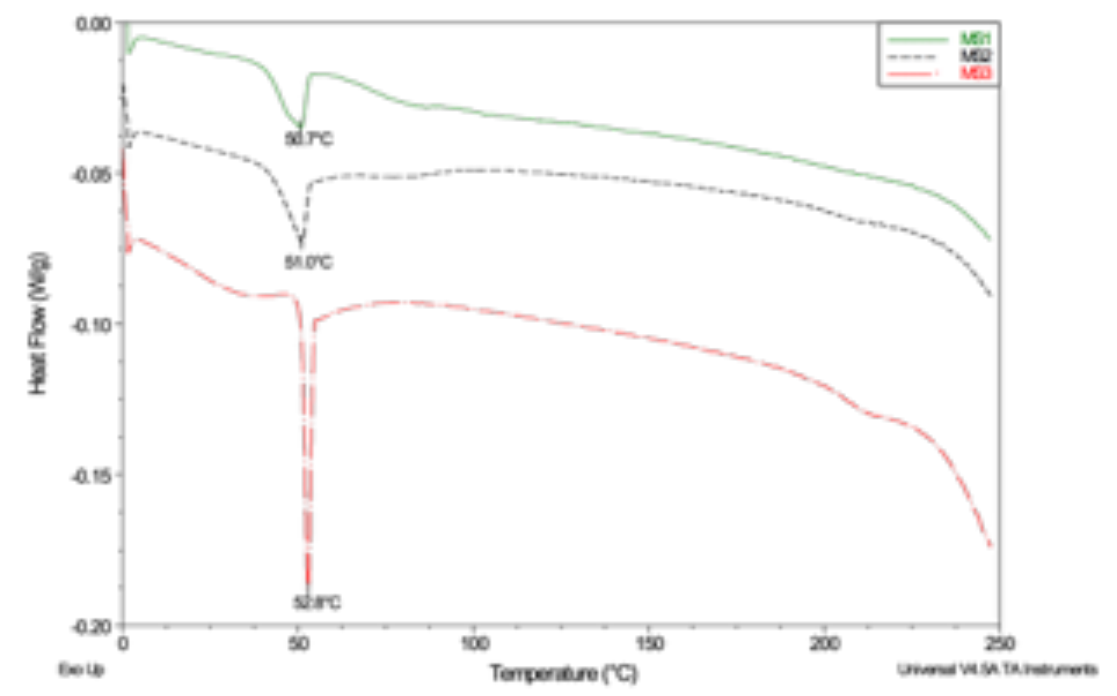

Figure 9 MTDSC heat flows of BSA loaded PLGA microspheres with three levels of drug loading

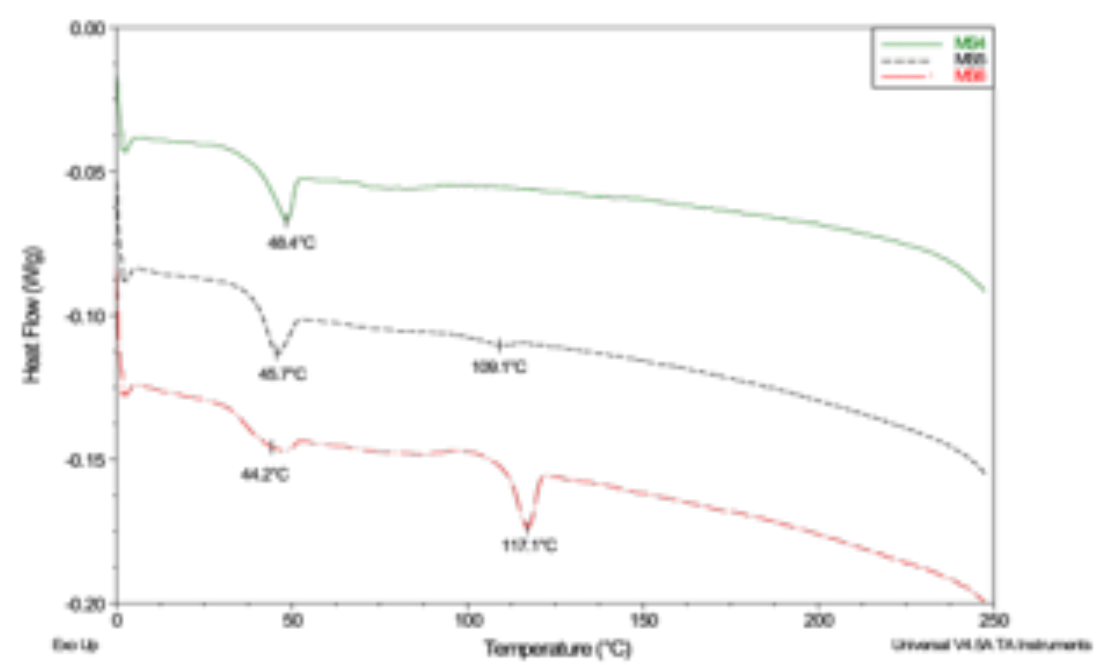

Figure 10 MTDSC heat flows of nimodipine loaded PLGA microspheres with three levels of drug loading

\subsection{TTM results}

Transition temperature microscopy (TTM) is a relatively new technique which involves mapping a sample surface via the temperature at which a heated probe indents into that surface, thereby effectively creating a map of softening points. The 
TTM color coded maps shown here correspond to an area of $50 \times 50 \mu \mathrm{m}$ for each sample. The corresponding histogram is a summary of the measured penetration temperatures. The temperature peak with the greatest intensity in the histogram corresponds to the most prevalent component/phase in the sample.

TTM was firstly used to analyze the raw materials of BSA, nimodipine and PLGA. The results were shown in Figure 11. In the transition map of BSA, it was shown that BSA had several different thermal transitions which were expressed as different characteristic color blocks. It had previously been shown that BSA consisted of peptides of different secondary structures: $50-68 \%$ of which are $\alpha$-helix peptides, and $16-21 \% \beta$-sheet $[24,25]$. Characteristic peaks appeared at multiple temperature points in the histogram of BSA, and the strongest response was concentrated in the region above $100{ }^{\circ} \mathrm{C}$, which could be used to analyze BSA qualitatively in the later experiment.

The thermal transition map of nimodipine consists largely of a single characteristic color. Nimodipine is a crystalline small molecule drug that has high purity when chemically synthesized and therefore it has a defined melting point. These material characteristics account for the single color observed in the thermal transition map as well as the comparatively narrow distribution of thermal transitions in the histogram for this material. Specifically, the transitions occurred in the range of $60-90{ }^{\circ} \mathrm{C}$, with the highest frequency occurring at approximately $80^{\circ} \mathrm{C}$.

The thermal transition map of the PLGA raw material was also fairly consistent. The histogram shows that the characteristic thermal transition peaks of PLGA occur at 50-55 ${ }^{\circ} \mathrm{C}$. These findings agreed well with the value measured using DSC, i.e. $48.1^{\circ} \mathrm{C}$.

After the qualitative analysis of the three substances individually, drug distribution on the surface and cross-sections of both BSA-PLGA and nimodipinePLGA microspheres with different drug loadings were analyzed with TTM. Figure 12 showed that due to the great difference of the transition temperatures between BSA and PLGA, the distribution of BSA on the surface and internal cross-section of the 
BSA-PLGA microspheres could be clearly seen. In the transition maps of the BSAPLGA microspheres, the transitions occurring at a lower temperature, represented by blue blocks, were the areas of PLGA; while the transitions occurring at a higher temperature, represented by bright green blocks, were the areas of BSA. The transition maps of both the surfaces and the cross-sections of the microsphere formulations showed that when the BSA loading is increased from $3.66 \%$ to $8.84 \%$, there was a clear increase in the characteristic colors of BSA on the transition maps. Additionally, the quantity and intensity of the peaks on the histograms in the region above $100{ }^{\circ} \mathrm{C}$ (which can be attributed to BSA thermal transitions) increased with increasing BSA load. However, it must be noted that the actual drug loadings $(3.66 \%$ and $8.84 \%$ ) measured using chemical method were determined on the bulk samples while TTM was mapping the drug distribution on the localized surface regions of a single microsphere. Therefore, the size of the total areas of a specific color in the TTM map would not necessarily reflect on the overall drug loading.

Comparing the TTM results of the surfaces and cross-sections of the microspheres with equal drug loading, it was found that there was more area covered by the color blocks associated with BSA inside the microsphere than on the surface. As would be expected, the quantity and intensity of the peaks associated with BSA in the histograms produced of the transition temperatures of the microsphere crosssection were all higher than those in the histograms of microsphere surface. This TTM data suggested that for BSA-PLGA microspheres of equal drug loading, more BSA was distributed on the interior of the microspheres than on the surface.

The TTM results of nimodipine microspheres are shown in Figure 13. The results of the raw material characterization showed that the transition temperatures of PLGA and nimodipine determined by TTM were very close, which means that the characteristic color blocks of the two components were very similar. Therefore, with the nimodipine-PLGA microspheres, the transition temperature maps largely consisted of a single color block and it was very difficult to distinguish the areas corresponding to nimodipine and PLGA and also hard to monitor the distribution of nimodipine on the surface and cross-section of PLGA microspheres. It is suggested 
that TTM technique is not able to monitor microstructure/drug distribution with all microsphere formulations. A critical criterion/limitation for applying this technique is that there must be a marked difference between the thermal transition temperatures of each individual components/phases in the formulation. For instance, the thermal transitions of nimodipine rich region and PLGA rich region were at similar temperatures hence overlapping characteristic color blocks in the TTM map, which complicated the differentiation. In terms of the magnitude of such difference, it is indicated in this study that it should be at least over circa $40^{\circ} \mathrm{C}$ to generate distinct colors in TTM map. It was also interesting to find some round dark pink color areas in a majority of TTM maps, which could most probably be a cavity/gap region. When the probe $(30 \mathrm{~nm}$ in diameter) landed at a cavity point, there was no contact between the probe and the material and this is normally processed by the software as a low $\left(0^{\circ} \mathrm{C}\right)$ temperature reading. This has also been seen in other investigations by our group.

Due to the limitation of using TTM color coded map discussed above, the histograms produced from the transition temperatures can be used to obtain more information about the loading of the different components. The temperature peak with the greatest intensity in the histogram corresponds to the most prevalent component/ phase in the sample. In terms of the nimodipine-PLGA microspheres in this study, at the transition temperatures near $80{ }^{\circ} \mathrm{C}$, where the transitions associated with nimodipine appeared, the quantity and intensity of the histogram peaks increased with increasing nimodipine load. Furthermore, when comparing the histograms of the surfaces and cross-sections of nimodipine microspheres, both the quantity and intensity of the characteristic peaks of nimodipine in the histograms of the microsphere cross-sections were higher than those in the histograms of the microsphere surface. These results indicated that when nimodipine-PLGA microspheres were prepared by the membrane-emulsification method, more nimodipine was present in the interior of the microsphere than on the surface. This relationship was more obvious in the TTM results of the nimodipine microspheres with the higher drug loading of $22.80 \%$. At the lower nimodipine loading of $8.30 \%$ 
the histogram peaks associated with the transition temperature of nimodipine were fewer, making this relationship indistinctive.

In terms of the two thermal analysis approaches used in this study, i.e. MTDSC and TTM, MTDSC measures all the thermal transitions of the bulk sample while TTM only picks up the first significant response to the increased temperature at each data point within a small local region of the sample, including thermal transitions (e.g. melting, glass transition) as well as any other responses which involves probe sinking in, such as simply sample softening. In this case, there is always a difference in temperature measured between MTDSC and TTM. The pattern of variation normally depends on the nature of the sample under investigation, rather than following a definitive trend. This explains the different variations observed with BSA and nimodipine samples. Comparatively, there was a greater variation associated with BSA as compared to nimodipine. This was mainly due to the larger and more complex structure of BSA. However, we should note that the first thermal transition of BSA measured using MTDSC (Fig. 8(a)) showed a very 'broad' peak with a maximum value at $62.5^{\circ} \mathrm{C}$ and an offset between $100-150^{\circ} \mathrm{C}$, which better agreed with the TTM observation, i.e. over $100^{\circ} \mathrm{C}$.
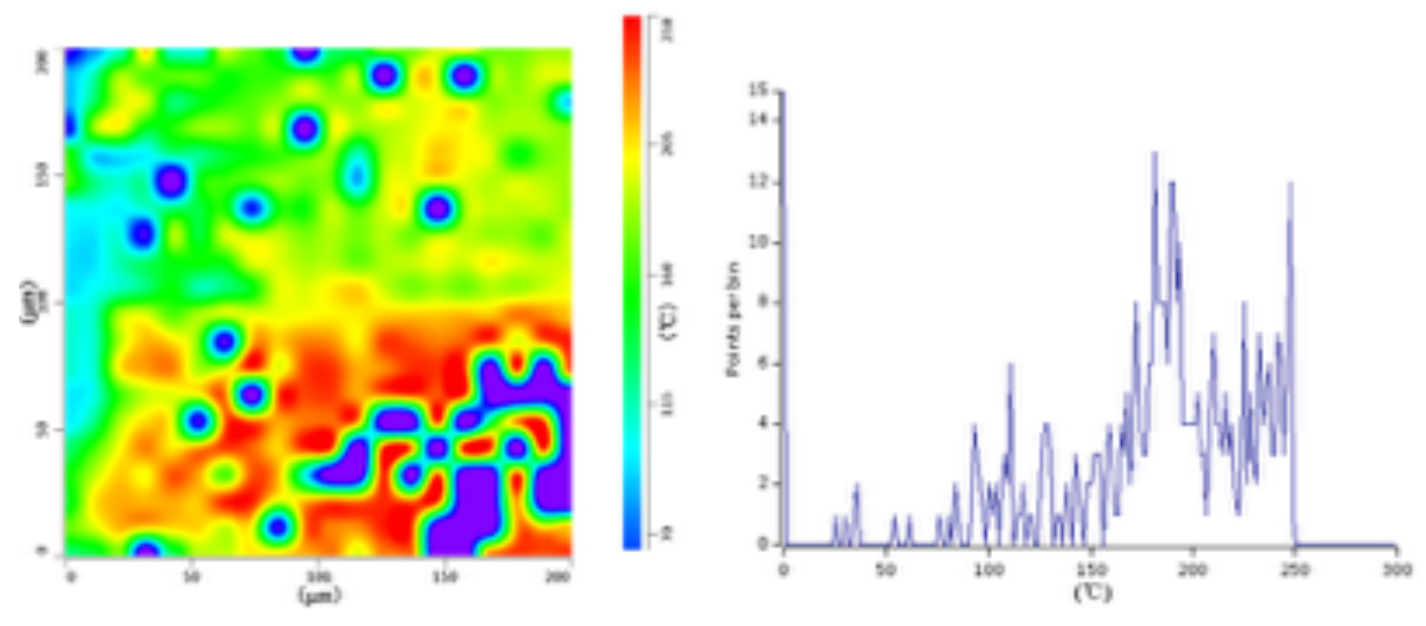

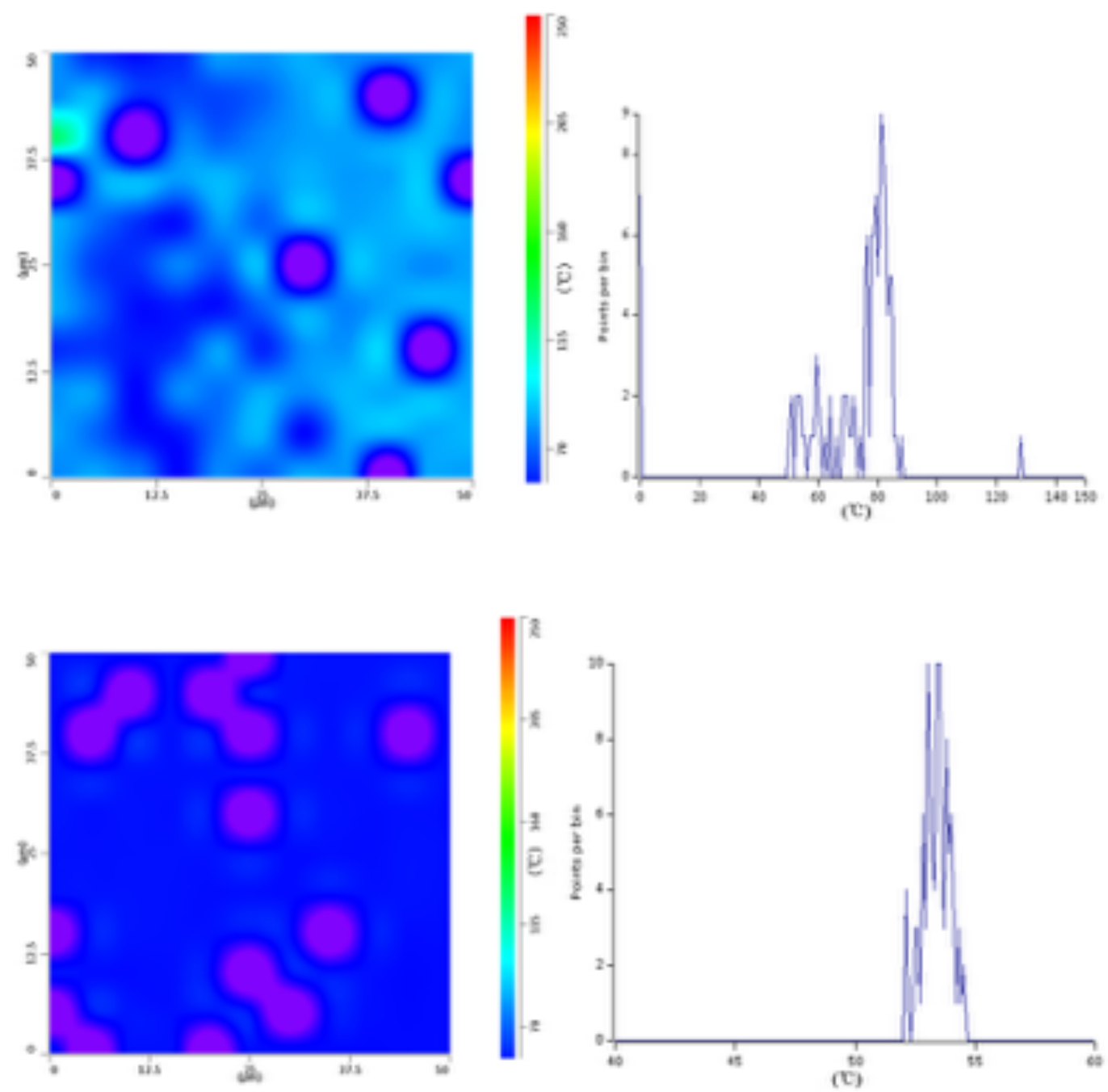

Figure 11 TTM color coded map (left) and corresponding histogram (right) of BSA, nimodipine and PLGA raw materials (top to bottom)
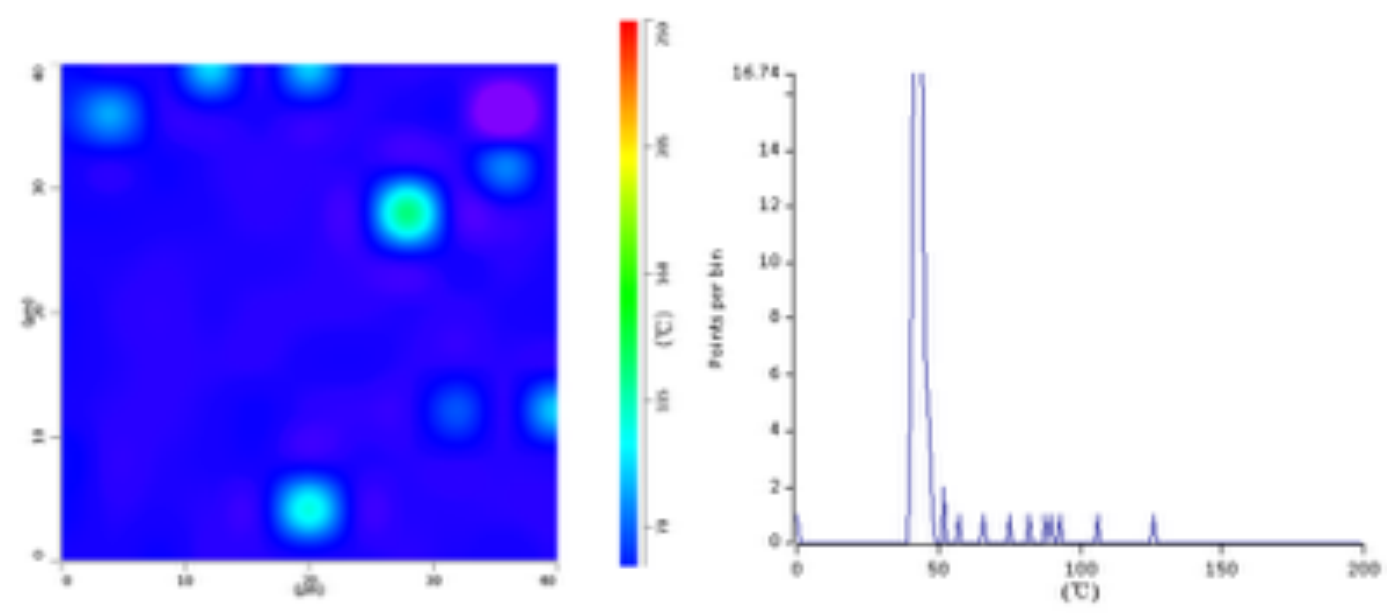

a. The outer surface of 3.66\% BSA loaded PLGA microspheres 

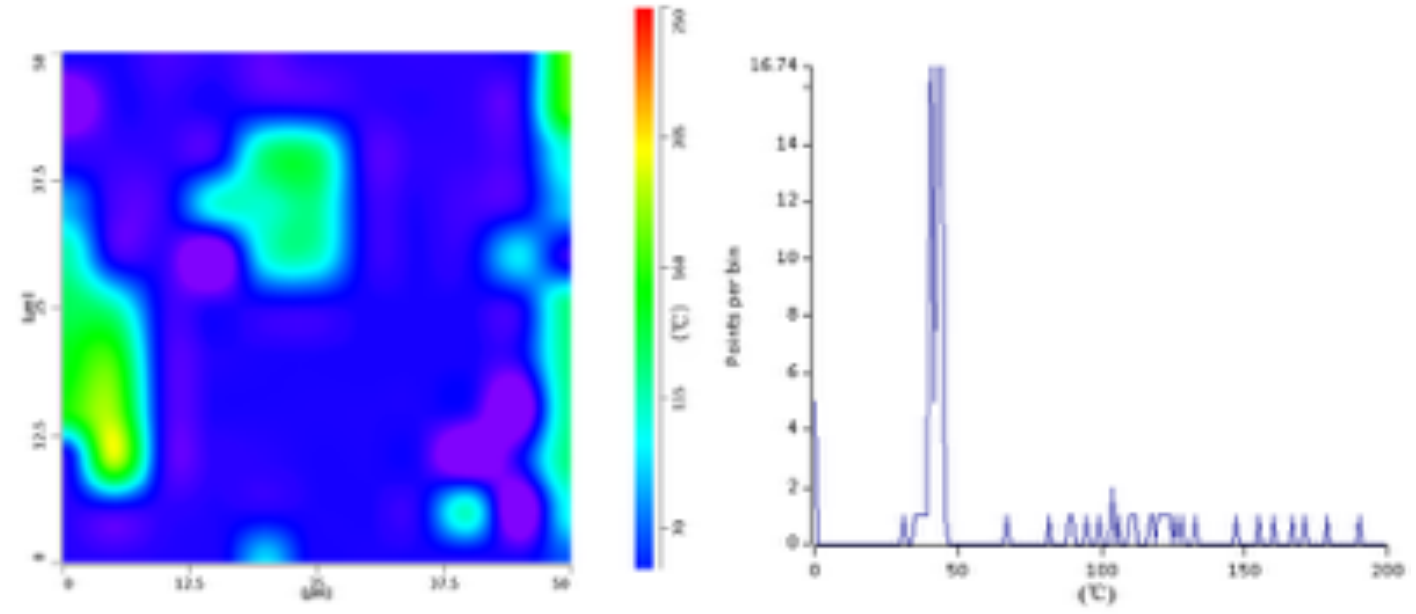

b. The outer surface of $8.84 \%$ BSA loaded PLGA microspheres
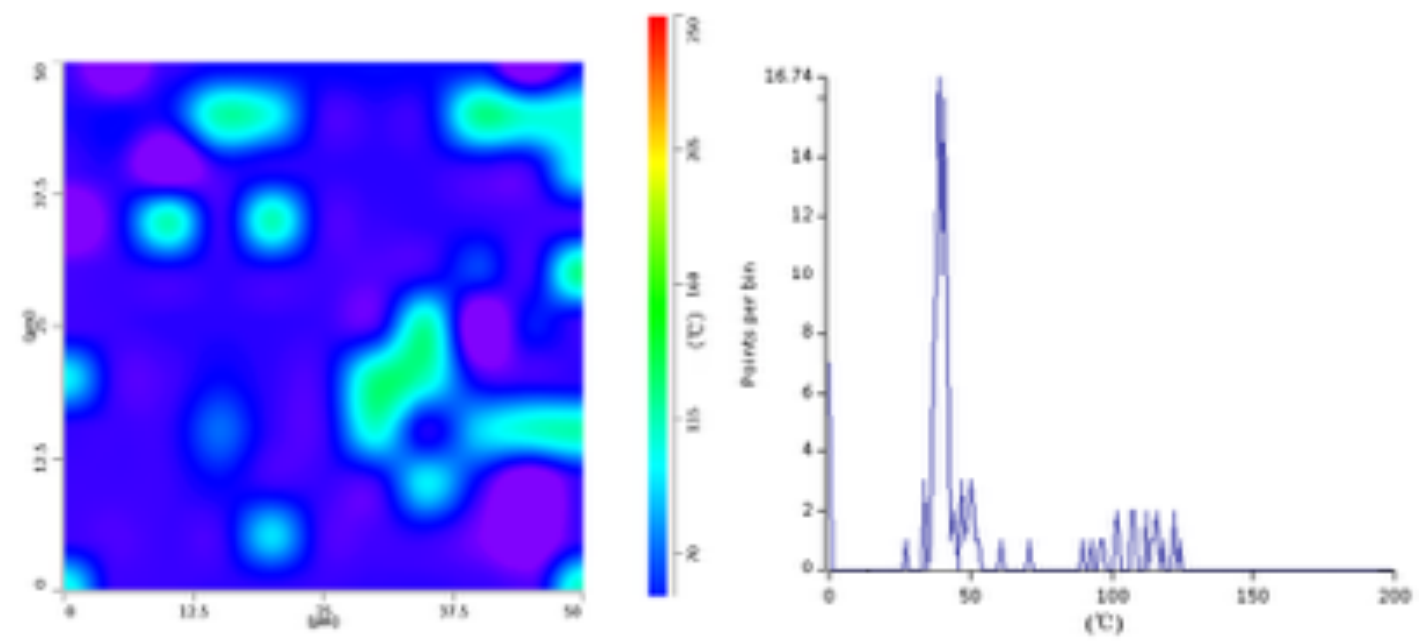

c. The cross section of $3.66 \%$ BSA loaded PLGA microspheres
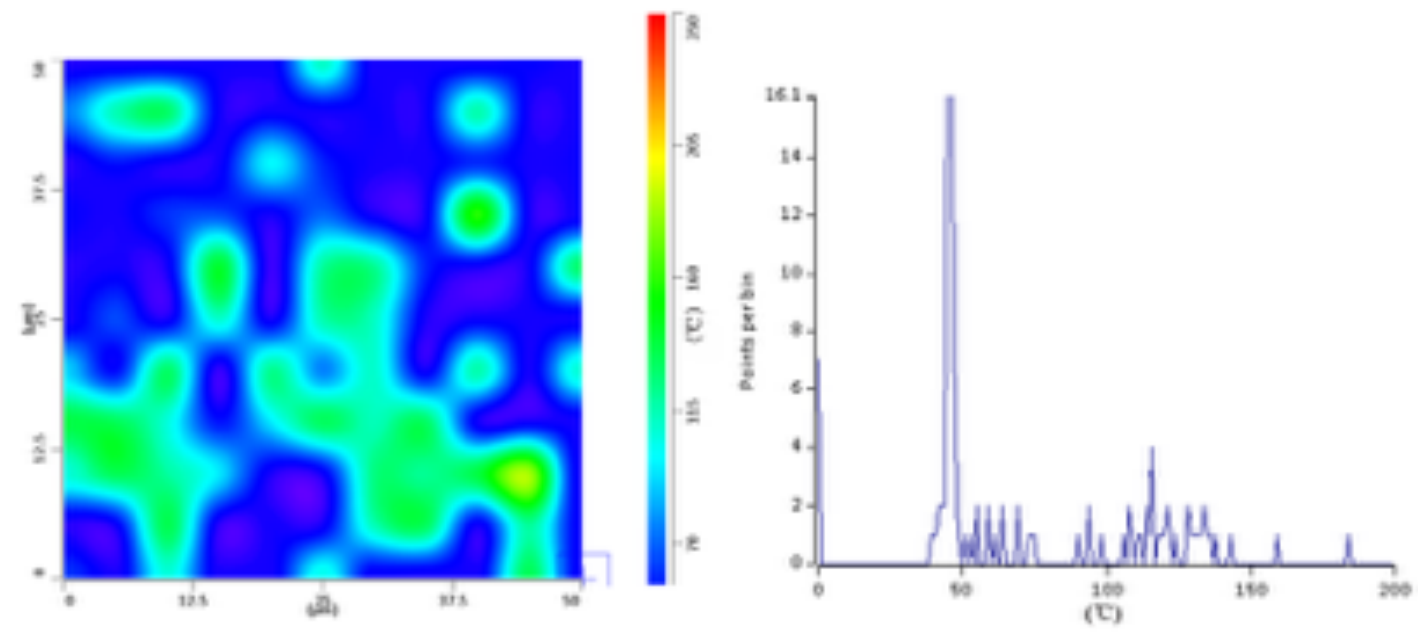

d. The cross section of $8.84 \%$ BSA loaded PLGA microspheres 
Figure 12 TTM color coded maps (left) and corresponding histograms (right) of the outer surface (a-b) and cross section (c-d) of BSA loaded PLGA microspheres with the lowest and highest drug loading
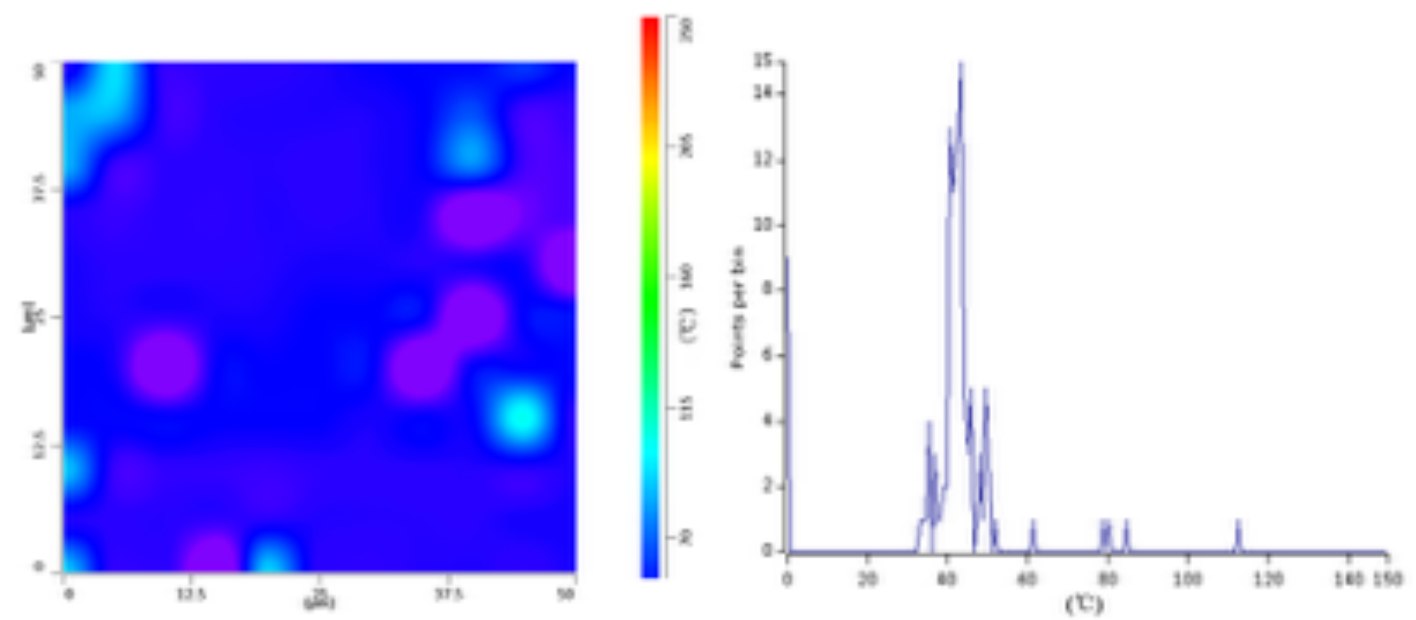

a. The outer surface of $8.30 \%$ nimodipine loaded PLGA microspheres
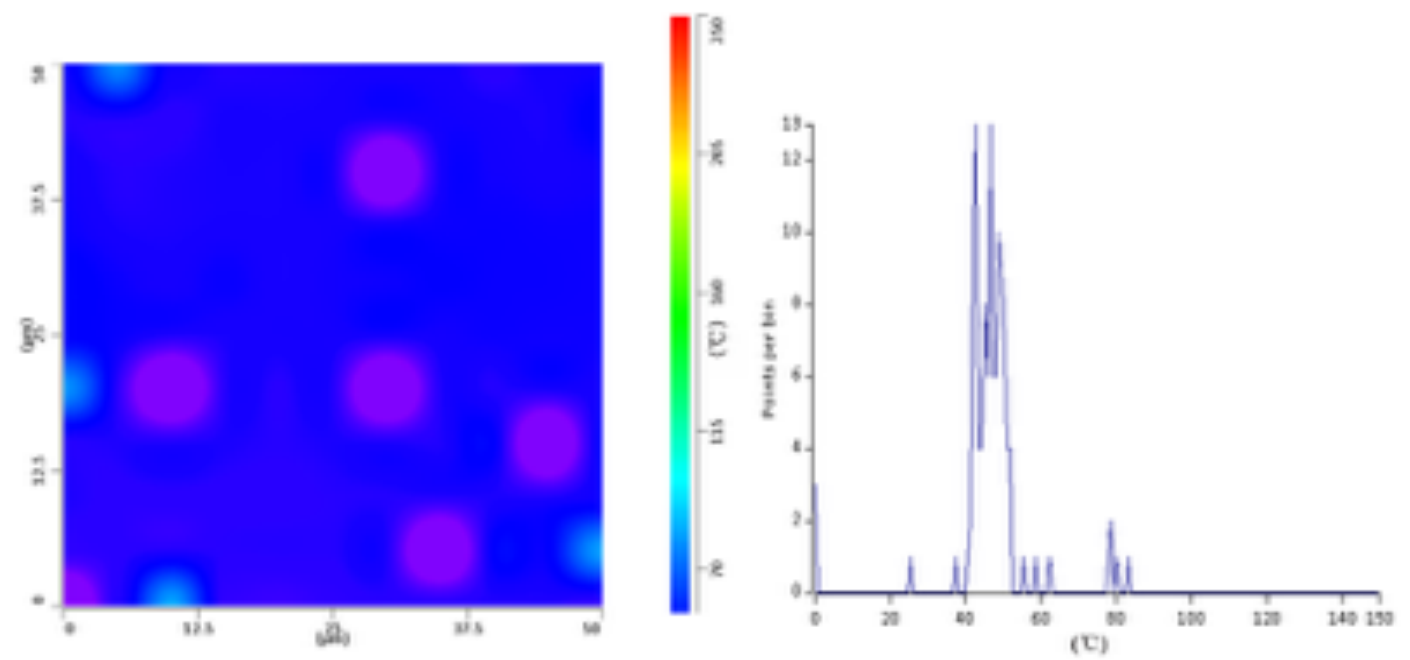

b. The outer surface of $\mathbf{2 2 . 8 0 \%}$ nimodipine loaded PLGA microspheres 

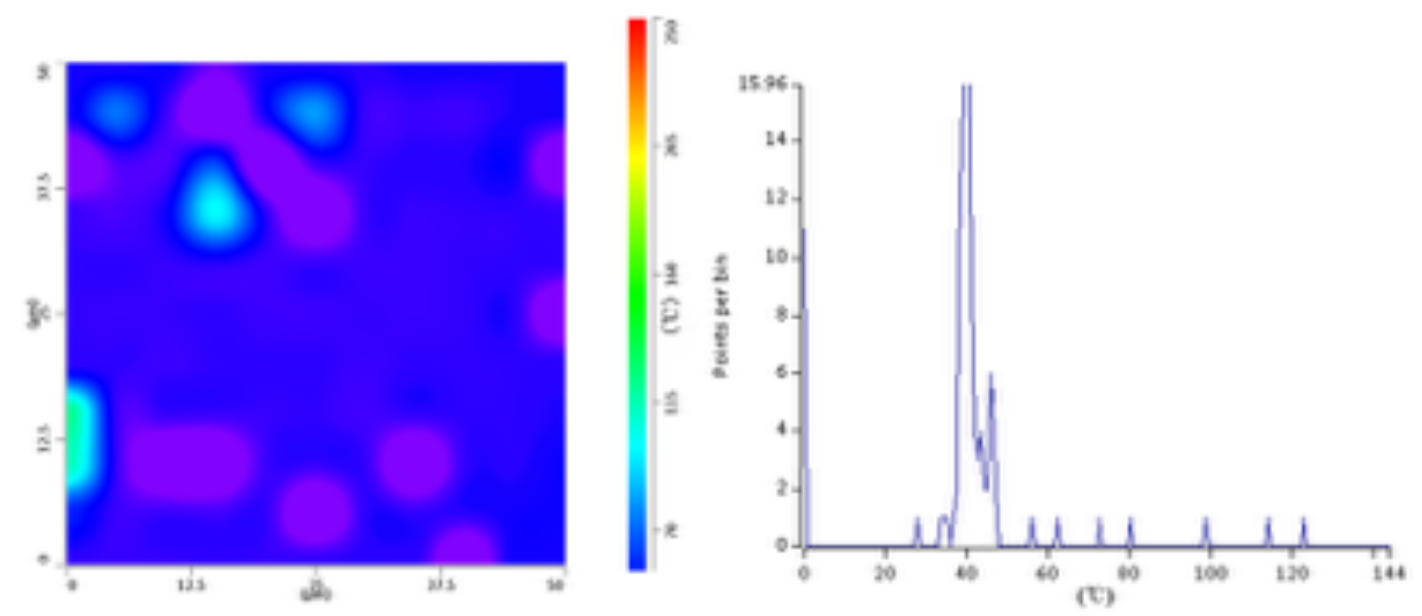

c. The cross section of $8.30 \%$ nimodipine loaded PLGA microspheres
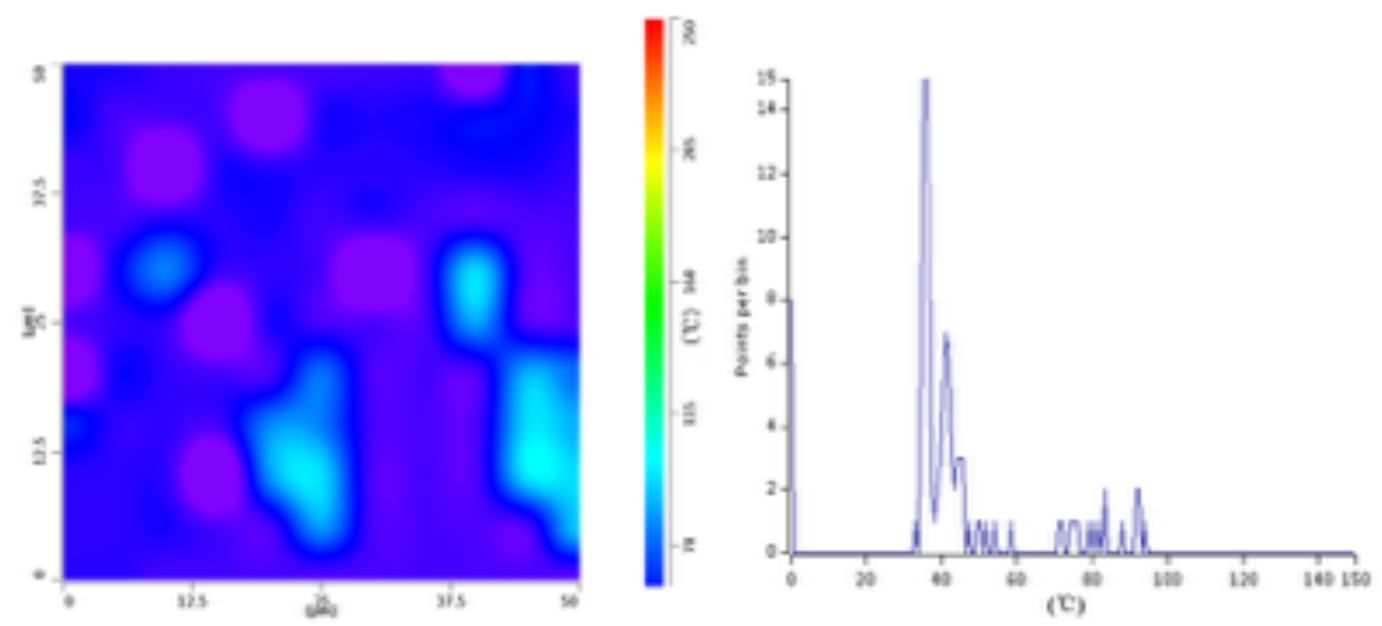

d. The cross section of $22.80 \%$ nimodipine loaded PLGA microspheres

Figure 13 TTM color coded maps (left) and corresponding histograms (right) of the outer surface (a-b) and cross section (c-d) of nimodipine loaded PLGA microspheres with the lowest and highest drug loadings.

\section{Discussion}

In this study several PLGA microsphere formulations produced with two different model drugs and preparation methods were extensively characterized with SEM, XRD, FTIR, MTDSC and TTM. SEM was able to show differences in surface and internal morphology of the microsphere formulations produced by two different preparation methods. XRD was employed to analyze the physical state of the drugs 
encapsulated in the microspheres, while FTIR allowed analysis of the molecular structure of the drugs in microspheres. MTDSC was able to analyze the bulk thermodynamic properties of the microsphere products. TTM, meanwhile, is a spatially resolved thermal analysis technique that was applied in the study to analyze the surface and cross-sections of individual microspheres.

Since the encapsulated drug and PLGA polymer had their own thermal properties, the corresponding color blocks of the different components shown in maps were also different. As a result, the loading and distribution of a certain substance in a selected area could be easily determined by observing the thermal transition maps, if the thermodynamic properties of the materials were significantly different. The role of the histograms in TTM was similar to MTDSC.

The results of TTM, however, were strongly influenced by the properties of the sample itself:

(1) The thermal transitions of nimodipine and PLGA occurred at a similar temperature which resulted in the characterstic color blocks also being very similar; therefore, when analyzing nimodipine-PLGA microspheres, it was not possible to determine the loading or distribution of the components from the TTM maps. When melting point difference of two substances is over $40^{\circ} \mathrm{C}$ TTM technology can be used to determine drug distribution.

The thermodynamic properties of BSA and PLGA were very different, however, and as a result it was possible to visually distinguish regions of BSA and PLGA on the TTM maps produced, allowing spatial distribution and BSA loading to be qualitatively assessed. It was noteworthy that, BSA showed a variety of color blocks in its own map probably because of containing the peptides with different secondary structures. However, in the thermal transition maps of the BSA microspheres, BSA was just shown by bright green color blocks, while the color blocks representing the higher temperature transitions (such as the yellow and red blocks) were not present. This phenomenon was reflected in the histograms of BSA microspheres. In the histogram of BSA raw material, the region where the quantity and intensity of the 
thermal transition peaks was highest was concentrated in the $150-200{ }^{\circ} \mathrm{C}$ region. The quantity and intensity of the thermal transition peaks appearing at the $100-150{ }^{\circ} \mathrm{C}$ region were lower than those in $150-200{ }^{\circ} \mathrm{C}$ region. However, when analyzing the histograms of the BSA microspheres, the thermal transition peaks of BSA mainly appeared in the lower range of $100-150{ }^{\circ} \mathrm{C}$.

The TTM results suggest that after being encapsulated into microspheres, the thermal transitions of BSA tended to occur at a lower temperature. The FTIR results showed that after being encapsulated most of the $\alpha$-helix peptides in BSA changed conformation to $\beta$-sheet peptides. This change in secondary structure was probably caused by the formation of hydrogen bonds with PLGA in the polymer matrix. Moreover, with the formation of hydrogen bonds, the two components transformed to a relatively stable "dispersed system", the thermal properties of which fell in between those of BSA and PLGA separately. More specifically, the transition temperatures of BSA-PLGA individual microspheres as determined by TTM presented a lower temperature as compared to BSA alone due to the contribution of the thermal properties of PLGA. Furthermore, it was also found that BSA within the PLGA matrix showed a significantly slower release rate although it has a high solubility. This was due to the hydrophilic nature of the drug as well as the hydrogen bonding formed between the drug and matrix in the dispersed system.

One can conclude from the findings above that TTM is a very promising technique for monitoring the microstructure of individual microspheres provided that all components in the formulation present a considerable difference in their thermal properties.

(2) The drug loading achieved in the nimodipine-PLGA microspheres was significantly higher than that achieved in the BSA-PLGA microspheres; however, in the histograms the thermal transition peaks associated with nimodipine were much lower than the peaks associated with BSA. Also, in the TTM maps of BSA microspheres, the area that was associated with BSA transitions was very large even though the drug loading was low. The differences in the structure and properties 
between nimodipine and BSA as well as their compatibility with PLGA as detailed below may explain this phenomenon.

Nimodipine is a small molecule drug, with a molecular weight of 418 and a simple spatial structure while PLGA is a long chain polymer. Due to the lipophilic nature of both materials, they present very good compatibility. The SEM images showed very few cavities in the cross-section of the nimodipine-PLGA microspheres, demonstrating a rather solid structure with such a formulation. The relatively high compatibility between nimodipine and PLGA as well as the stabilizing effect of PLGA on the relatively small molecules resulted in a well dispersed system, as reflected by XRD and MTDSC.

The molecular weight of the BSA (approximately $68 \mathrm{KDa}$ ) is much larger than nimodipine, and as a protein molecule, BSA has a spatial configuration with very complex secondary and tertiary conformations. These properties indicate that the same mass of BSA would occupy much larger space as compared to nimodipine. Its hydrophilic nature also makes it less compatible with PLGA. As a consequence, the SEM images showed many cavities inside the BSA microspheres due to the microspheres being prepared by double emulsion-solvent evaporation technique. The combination of MTDSC and XRD results demonstrated a fully amorphous structure with two separated phases for BSA-PLGA microspheres. This was most likely due to the incompatibility between BSA which is hydrophilic and water-soluble, and PLGA which is lipophilic and soluble in organic solvent. Therefore, once BSA was encapsulated within the microspheres it could only stay in the cavities of the PLGA matrix formed as a result of the double-emulsion production method, retaining its original independent phase. As a result, although the drug loading of BSA microspheres was low, the BSA distribution area shown in the TTM maps was relatively large as the system was phase-separated. To conclude, the differences in the chemical nature and solubilities of the components, and therefore the preparation method required for a formulation, would significantly affect the microstructure measured by TTM. 
(3) Comprehensive analysis of the results of TTM and the other analytical techniques increased the understanding of the spatial drug distribution within two different types of microspheres, and also contributed to the increased understanding of the in vitro drug release behaviors from the microspheres. The TTM results of the BSA-PLGA microsphere surface showed a reasonable quantity of BSA was present at the surface of the formulation. BSA molecules present near the microsphere surface would quickly dissolve and diffuse into the bulk aqueous release medium resulting in the burst release effect observed. However, the TTM maps also showed that the BSA present on the surface of the microspheres was just a small part of the total drug content as the TTM maps of the microsphere cross-sections showed that more BSA appeared to be present inside the microspheres. Therefore, despite the significant burst release, the majority of the BSA was distributed inside the microspheres and was not so easily released. The TTM results and in vitro release studies supported each other well for the BSA-PLGA microsphere formulations.

Due to the good chemical compatibility between nimodipine and PLGA, nimodipine could be distributed uniformly in the amorphous state throughout the PLGA matrix in the nimodipine-PLGA microspheres. The TTM results supported this proposed distribution, and the in vitro release studies show that nimodipine was released with a relatively constant rate as the PLGA matrix degraded. Therefore, the TTM results and in vitro release studies were again in agreement, and the TTM maps were able to help better understand the drug release profiles from the microsphere formulations.

\section{Conclusions}

In conclusion, TTM is a novel thermal and imaging technique that can be applied to study the spatial drug distribution of as single microspheres in relation to their in vitro drug release performance, especially for formulations consisting of components with significantly different thermal properties. By using TTM in combination with other techniques such as XRD, FTIR and MTDSC, the physical and chemical 
properties of microspheres could be understood more comprehensively as the different techniques complement each other.

\section{Acknowledgements}

We wish to thank the Guangdong Provincial Science and Technology Project (2013B021800091 and 2011A080504003) for funding. And the authors would also like to thank Goh Choon Fu and Awis Sukarni Mohmad Sabereb for their assistance with TTM.

\section{References}

[1] Cao H; Chen MM; Liu YY; Huang YQ; Wang JH; Chen JD; Zhang QQ. Fish collagen-based scaffold containing PLGA microspheres for controlled growth factor delivery in skin tissue engineering. Colloids Surf B Biointerfaces. 2015,136, 1098-1106.

[2] Dong H; Tang G; Ma T; Cao X. One-step fabrication of inorganic/organic hybrid microspheres with tunable surface texture for controlled drug release application. J Mater Sci Mater Med. 2016, 27, 7.

[3] Fang K; Song L; Gu Z; Yang F; Zhang Y; Gu N. Magnetic field activated drug release system based on magnetic PLGA microspheres for chemo-thermal therapy. Colloids Surf B Biointerfaces. 2015, 136, 712-720.

[4] Xie XG; Zhang M; Dai YK; Ding MS; Meng SD. Combination of vascular endothelial growth factor-loaded microspheres and hyperbaric oxygen on random skin flap survival in rats. Exp Ther Med. 2015, 10, 954-958.

[5] Zhao Q; Li ZY; Zhang ZP; Mo ZY; Chen SJ; Xiang SY; Zhang QS; Xue M. Polylactic-co-glycolic acid microspheres containing three neurotrophic factors promote sciatic nerve repair after injury. Neural Regen Res. 2015, 10, 1491-1497.

[6] Zhou C; Cui D; Zhang Y; Yuan H; Fan T. Preparation and characterization of 
ketoprofen-loaded microspheres for embolization. J Mater Sci Mater Med. 2012, $23,409-418$.

[7] Cui DC; Lu WL; Sa EA; Gu MJ; Lu XJ; Fan TY. Poly( acrylic acid) microspheres loaded with lidocaine: preparation and characterization for arterial embolization. Int J Pharm. 2012, 436, 527-535.

[8] Sareen R; Jain N; Rajkumari A; Dhar KL. pH triggered delivery of curcumin from Eudragit-coated chitosan microspheres for inflammatory bowel disease: characterization and pharmacodynamic evaluation. Drug Deliv. 2016, 23, 55-62.

[9] Gan L; Gao YP; Zhu CL; Zhang XX; Gan Y. Novel pH-sensitive lipid-polymer composite microspheres of 10-hydroxycamptothecin exhibiting colon-specific biodistribution and reduced systemic absorption. J Pharm Sci. 2013, 102, 1752-1759.

[10] Lagarce F; Cruaud O; Deuschel C; Bayssas M; Griffon-Etienne G; Benoit J. Oxaliplatin loaded PLAGA microspheres: design of specific release profiles. Int J Pharm. 2002, 242, 243-246.

[11] Floyd JA; Galperin A; Ratner BD. Drug encapsulated polymeric microspheres for intracranial tumor therapy: A review of the literature. Adv Drug Deliv Rev. $2015,91,23-37$.

[12] Lin X; Xu Y; Tang X; Zhang Y; Chen J; Zhang Y; He H; Yang Z. A uniform ultrasmall microsphere/SAIB hybrid depot with low burst release for long-term continuous drug release. Pharm Res, 2015, 32, 3708-3721.

[13] Fan Yang, Yajun Shu, Yiqun Yang, Fenglan Song, Yufang Pan, Xiaoying Long, Gang Chen, Yongming Zhang. The pharmacokinetics of recombinant human Interferon-alpha-2b poly(lactic-co-glycolic acid) (PLGA) microspheres in rats.Journal of Microencapsulation,2011,28(6):483-489.

[14] Ekaterina Lengert, Alexey M. Yashchenok, Vsevolod Atkin, Ales Lapanje, Dmitry A. Gorin, Gleb B. Sukhorukov and Bogdan V. Parakhonskiy. Hollow silver alginate microspheres for drug delivery and surface enhanced Raman scattering detection. RSC Adv., 2016, 6, 20447-20452.

[15] Manli Wang, Xiaolong Lu, Xianzhen Yin, Yajun Tong, Weiwei Peng, Li Wu, Haiyan Li, Yan Yang, Jingkai Gu, Tiqiao Xiao, Min Chen, Jiwen Zhang. 
Synchrotron radiation-based Fourier-transform infrared spectromicroscopy for characterization of the protein/peptide distribution in single microspheres. Acta Pharmaceutica Sinica B 2015;5(3):270-276.

[16] Hammiche A; Hourston DJ; Pollock HM. Scanning thermal microscopy: subsurface imaging, thermal mapping of polymer blends, and localized calorimetry. J Vac Sci Technol B. 1996,14, 1486-1491.

[17] Kim KJ; Park K; Lee J. Naonotopographical imaging using a heated atomic force microscope cantilever probe. Sensors Actuators A Phys. 2007,136, 95-103.

[18] Pollock HM; Hammiche A. Micro-thermal analysis: techniques and applications. J Phys D Appl Phys. 2001, 34, R23-53.

[19] Moffat JG; Qi S; Craig DQ. Spatial characterization of hot melt extruded dispersion systems using thermal atomic force microscopy methods: The effects of processing parameters on phase separation. Pharm Res. 2014, 31, 1744-1752.

[20] Dai X, Moffat JG, Wood J, Reading M. Thermal scanning probe microscopy in the development of pharmaceuticals. Adv Drug Del Rev. 2012, 64, 449-460.

[21] Li N; Mai HY; Luo YY. Study on cryo-sectioning technique of poly (lactic-coglycolic acid) microspheres. Journal of Guangdong Pharmaceutical University. $2014,30,1-5$.

[22] Kuznetsov AN; Ebert B; Lassmann G; Shapiro AB. Adsorption of small molecules to bovine serum albumin studied by the spin-probe method. Biochim Biophys Acta. 1975, 379, 139-146.

[23] Lin VJC.; Koenig JL. Raman studies of bovine serum albumin, Biopolymers. $1976,15,203-218$.

[24] Reed RG; Feldhoff RC; Clute OL; Peters T Jr. Fragments of bovine serum albumin produced by limited proteolysis. Conformation and ligand binding. Biochemistry. 1975,14, 4578-4583.

[25] Antonov YA; Wolf BA. Calorimetric and structural investigation of the interaction between bovine serum albumin and high molecular weight dextran in water. Biomacromolecules. 2005, 6, 2980-2989. 